\title{
A TLR9-dependent checkpoint governs B cell responses to DNA-containing antigens
}

\author{
Vishal J. Sindhava, ${ }^{1}$ Michael A. Oropallo, ${ }^{1}$ Krishna Moody, ${ }^{2,3}$ Martin Naradikian, ${ }^{1}$ Lauren E. Higdon, ${ }^{1}$ Lin Zhou, ${ }^{1,4}$ Arpita Myles, ${ }^{1}$ \\ Nathaniel Green, ${ }^{2,3}$ Kerstin Nündel, ${ }^{3}$ William Stohl, ${ }^{5}$ Amanda M. Schmidt, ${ }^{1}$ Wei Cao, ${ }^{6}$ Stephanie Dorta-Estremera, ${ }^{6}$ \\ Taku Kambayashi, ${ }^{1}$ Ann Marshak-Rothstein, ${ }^{3}$ and Michael P. Cancro ${ }^{1}$ \\ 'Department of Pathology and Laboratory Medicine, University of Pennsylvania, Perelman School of Medicine, Philadelphia, Pennsylvania, USA. ²Department of Microbiology, Boston University \\ School of Medicine, Boston, Massachusetts, USA. ${ }^{3}$ Department of Medicine, University of Massachusetts School of Medicine, Worcester, Massachusetts, USA. ${ }^{4}$ Department of Laboratory Medicine, \\ Shanghai Changzheng Hospital, Second Military Medical University, Shanghai, China. ${ }^{5}$ Division of Rheumatology, Department of Medicine, University of Southern California Keck School of Medicine, \\ Los Angeles, California, USA. ${ }^{6}$ Department of Immunology, University of Texas MD Anderson Cancer Center, Houston, Texas, USA.
}

\begin{abstract}
Mature B cell pools retain a substantial proportion of polyreactive and self-reactive clonotypes, suggesting that activation checkpoints exist to reduce the initiation of autoreactive $B$ cell responses. Here, we have described a relationship among the B cell receptor (BCR), TLR9, and cytokine signals that regulate B cell responses to DNA-containing antigens. In both mouse and human B cells, BCR ligands that deliver a TLR9 agonist induce an initial proliferative burst that is followed by apoptotic death. The latter mechanism involves p38-dependent $G_{1}$ cell-cycle arrest and subsequent intrinsic mitochondrial apoptosis and is shared by all preimmune murine B cell subsets and CD27- human B cells. Survival or costimulatory signals rescue B cells from this fate, but the outcome varies depending on the signals involved. B lymphocyte stimulator (BLyS) engenders survival and antibody secretion, whereas CD40 costimulation with IL-21 or IFN- $\gamma$ promotes a T-bet ${ }^{+}$B cell phenotype. Finally, in vivo immunization studies revealed that when protein antigens are conjugated with DNA, the humoral immune response is blunted and acquires features associated with T-bet ${ }^{+}$B cell differentiation. We propose that this mechanism integrating BCR, TLR9, and cytokine signals provides a peripheral checkpoint for DNA-containing antigens that, if circumvented by survival and differentiative cues, yields B cells with the autoimmune-associated T-bet ${ }^{+}$phenotype.
\end{abstract}

\section{Introduction}

Despite the elimination of many autoreactive B cells during development $(1,2)$, mature B cell pools include a substantial proportion of polyreactive and self-reactive clonotypes (3-5). This observation suggests that later, activation-associated checkpoints exist to minimize the likelihood that such cells will engage in antibody production, memory B cell formation, or affinity maturation focused on self-antigens. Several recent observations bear directly on this possibility. First, mounting evidence indicates that neither the presence nor the activation of these autoreactive clones is sufficient to engender autoantibody production; instead, additional signals are needed to overcome regulatory constraints that prevent frank autoimmunity (6-14). Cognate $\mathrm{T}$ cell help, B lymphocyte stimulator (BLyS, also known as BAFF), IFN- $\gamma$, and IL-21 have been implicated as possible second signals (15-25). BLyS overexpression yields humoral autoimmunity (13), and both IFN- $\gamma$ and IL-21 play roles in systemic autoimmune diseases (26-29). Second, many autoantibodies bind DNA- or RNA-containing complexes, and numerous studies link the endosomal nucleic acid-sensing receptors TLR9 and TLR7 to autoimmune diseases $(12,13,15,18,30-34)$. Surpris-

Authorship note: V.J. Sindhava and M.A. Oropallo contributed equally to this work. Conflict of interest: The authors have declared that no conflict of interest exists. Submitted: August 8, 2016; Accepted: January 26, 2017.

Reference information: J Clin Invest. 2017;127(5):1651-1663.

https://doi.org/10.1172/JCl89931. ingly, TLR9 deficiency exacerbates autoimmune symptoms in several mouse models, indicating that TLR9 may play a role in limiting the activation of autoreactive B cells. Finally, recent evidence ties this signaling triad $-\mathrm{B}$ cell receptor (BCR), TLR7/9, and IL-21 or IFN- $\gamma$ - to the generation of T-bet ${ }^{+} \mathrm{CD} 11 \mathrm{c}^{+} \mathrm{B}$ cells (35), which are associated with autoimmunity in both mice and humans $(36,37)$. Together, these observations suggest a relationship among the BCR, TLR9, and cytokines that govern both normal and self-reactive antibody responses to nucleic acid-containing antigens, but the nature of this tripartite interaction remains unclear.

Herein, we show that in both mouse and human B cells, TLR9 agonists linked to BCR ligands induce apoptotic death after an initial proliferative burst. The underlying mechanism involves p38 MAPK-dependent cell-cycle arrest, followed by intrinsic mitochondrial apoptosis. However, B cells undergoing this program can be rescued, and the mode of rescue determines subsequent B cell fate. Whereas BLyS affords differentiation to antibody secretion, CD40 costimulation with either IFN- $\gamma$ or IL-21 yields the T-bet ${ }^{+} \mathrm{B}$ cell phenotype. Finally, we show in vivo that when antigens are complexed with DNA, the magnitude and quality of humoral responses are altered. Together, these findings reveal a cell-intrinsic, TLR9-dependent mechanism that governs the initiation, quality, and extent of $\mathrm{B}$ cell responses to DNA-associated antigens. Further, our data suggest that breaching this checkpoint may provide a route to autoimmunity in the context of DNA-containing self-antigens. 


\section{Results}

DNA immune complexes induce self-limiting $B$ cell responses that are rescued by $B L y S$. Prior studies showed that rheumatoid factortransgenic (RF-transgenic) B cells from AM14 mice proliferate in a TLR9-dependent manner when stimulated with chromatin immune complexes (ICs) formed by the monoclonal antibody PL23 (38). To reconcile these findings with exacerbated autoimmune disease in $\mathrm{Tlr}^{-1-}$ mice, we performed analyses of cell division and survival under varying conditions. In these experiments, we used $\mathrm{CD}_{23}{ }^{+}$splenic B cells, which are $95 \%$ or more quiescent follicular (FO) B cells. Either BCR cross-linking with $\mathrm{F}\left(\mathrm{ab}^{\prime}\right)_{2}$ fragments of rabbit anti-mouse IgM (anti- $\mu$ ) or TLR9 stimulation with the oligodeoxynucleotide 1826 (ODN 1826) induced several rounds of division, with the majority of cells remaining alive (Figure 1A). We observed similar results in cells stimulated with a combination of ODN 1826 and anti- $\mu$. In contrast, proliferation induced by PL2-3 ICs was followed by overwhelming cell death (Figure 1A). This did not reflect nutrient exhaustion, since replenishing chromatin-ICstimulated cultures with fresh medium had no ameliorating effect. Strikingly, BLyS rescued the chromatin-IC-stimulated B cells, restoring viability at all time points (Figure 1, A and C).

To establish whether this response is characteristic of all $B$ cells, regardless of BCR specificity, we synthesized a stimulatory TLR9 immune complex (STIC9) consisting of a biotinylated CpG-rich dsDNA fragment of approximately $600 \mathrm{bp}$ derived from a murine genomic CpG island sequence, termed clone 11 (39), linked to biotinylated Fab fragments of rabbit anti-mouse IgM via streptavidin (SA) (Figure 1B, inset). In contrast to smaller, thioester-linked CpG oligonucleotides like ODN 1826, clone 11 cannot freely enter B cells but is transported to a TLR9 compartment by BCR-mediated internalization. Thus, STIC9 mirrors PL2-3 IC stimulation, but is independent of BCR specificity, involves only TLR9, and eliminates potential $\mathrm{Fc}-\gamma$ receptor engagement.

Splenic CD23+ B cells stimulated with STIC9 recapitulated the post-proliferative death and BLyS-mediated rescue seen with PL2-3-stimulated RF B cells (Figure 1B). A CpG-negative dsDNA IC (CGNEG) synthesized in a manner identical to our synthesis of STIC9 mimicked anti- $\mu$ alone, reflecting BCR cross-linking without concomitant TLR9 engagement (Figure 1B). We investigated the timing of post-proliferative death to allow the appropriate design of sampling points in subsequent mechanistic studies. The kinetics of post-proliferative death were similar in both PL2-3-stimulated RF-transgenic AM14 or STIC9-stimulated C57BL/6 B cells, commencing by 48 hours after stimulation and being virtually completed by 60 hours (Figure 1, C and D). Importantly, STIC9 and PL2-3 induced similar degrees of post-proliferative death in B cells from AM14 mice, indicating that STIC9 engages the key elements triggered by the natural autoantigen (Supplemental Figure 1A; supplemental material available online with this article; https:/doi. org/10.1172/JCI89931DS1). Moreover, B cells from several lupusprone strains - NZB/W F1, Sle1, Sle2, and Sle3 - showed similar post-proliferative death responses (Supplemental Figure 1, B and C). Thus, the breakthrough of autoantibody production in these models probably reflects inappropriate rescue and differentiation rather than an intrinsic defect in this mechanism per se.

The post-proliferative death response depends on TLR9 signals, since the proliferation and survival of $T \operatorname{lr} 9^{-/-} \mathrm{B}$ cells treated with STIC9 resembled anti- $\mu$-stimulated cells, reflecting BCR cross-linking in the absence of a TLR9 signal, despite internalization of the DNA CpG motif (Figure 1, E and F). Importantly, the death response induced by STIC 9 does not reflect substantial differences in BCR or TLR9 signal strengths, as the degrees of spleen-associated tyrosine kinase (SYK) phosphorylation and downstream nerve growth factor IB (Nur77) induction were similar in cultures stimulated with either STIC9 or anti- $\mu$ plus ODN 1826 (Figure 1, G and H). Moreover, ODN 1826 directly coupled to anti- $\mu$ mimicked STIC9, ruling out the possibility that differences in TLR9 binding valency or receptor avidity were responsible (Figure 1I). Thus, both natural and defined BCR ligands containing TLR9 agonists drive a unique program that abruptly terminates $B$ cell activation and expansion. Further, this self-limiting response is extended by survival-promoting signals like BLyS.

Post-proliferative death involves intrinsic mitochondrial cell death following p38-dependent cell-cycle arrest. We next interrogated the mechanisms of STIC9-induced B cell death. Post-proliferative death did not reflect trans effects, since PL2-3 had no effect on the survival of B6.SJL B cells cocultured with PL2-3-stimulated AM14 B cells (data not shown). Instead, we found that STIC9 directly induced apoptotic cell death, as revealed by caspase 9 and caspase 3 cleavage, which was blocked by BLyS (Figure 2A). Since apoptosis involves either a caspase 8-dependent extrinsic pathway or a caspase 8-independent intrinsic pathway, we asked which is initiated by STIC9. Because caspase 8-KO mice are embryonically lethal unless receptor-interacting protein kinase 3 (RIP3) is also absent (40), we compared STIC9-activated B cells from C57BL/6, $\mathrm{RIP3}^{-/}$, and caspase $8^{-/-} \mathrm{RIP}^{-/-}$mice. STIC9 induced equivalent cell death in the double-KO and control cell populations (Figure 2B), implicating the intrinsic apoptotic death pathway and attendant mitochondrial depolarization. BLyS-mediated rescue, as evidenced by blocked caspase 9 and 3 cleavage (Figure 2A), is consistent with death via this pathway, since BLyS sustains mitochondrial stability (41-43). Two BLyS receptors, BLyS receptor 3 (BR3, also known as BAFFR) and transmembrane activator and CAML interactor (TACI), are expressed on mature naive B cells, and either could account for the BLyS-mediated rescue $(44,45)$. STIC9 stimulation upregulated both BR3 and TACI (Supplemental Figure 2, A and B), but while rescue was intact in $\mathrm{Taci}^{-/-} \mathrm{B}$ cells, BLyS failed to rescue STIC9-stimulated BR3-deficient B cells (Supplemental Figure 2, C and D). Thus, BLyS-mediated rescue of post-proliferative death requires BR3, whereas TACI is dispensable. Consistent with the well-established ability of BR3 to induce BCL-XL and other antiapoptotic BCL-2 family members (43), B cells from BCL-XLtransgenic mice, which overexpress BCL-XL in the B cell lineage, resisted STIC9-mediated cell death, even in the absence of BLyS (Figure 2C). Finally, mitochondrial depolarization following STIC9 stimulation was confirmed by flow cytometric analyses and was prevented by BCL-XL overexpression (Figure 2C).

While these observations showed that intrinsic mitochondrial death is the ultimate route to TLR9-dependent post-proliferative death, the upstream initiating events remained unclear. Three MAPK mediators, the JNK, ERK, and p38 kinases, are integral to both BCR and TLR9 signaling $(46,47)$. MAPK signaling differs in AM14 B cells stimulated by PL2-3 versus those stimulated by anti- $\mu$ or ODN 1826 (48). Moreover, DNA-containing antigens 
A $\mathrm{AM} 14$

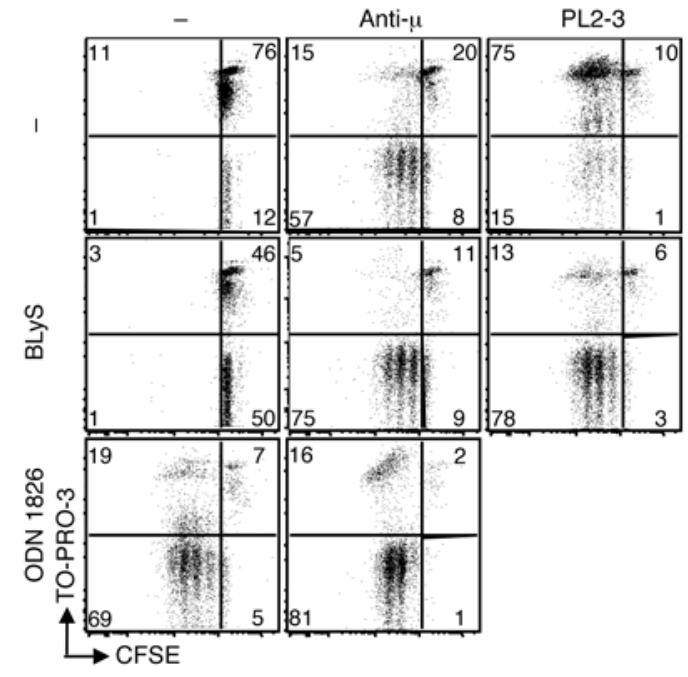

C

AM14

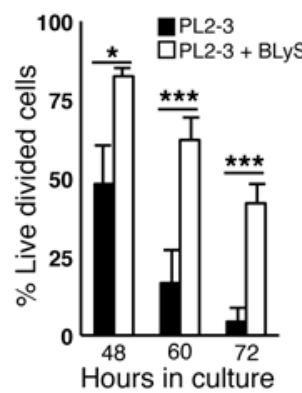

D

C57BL/6

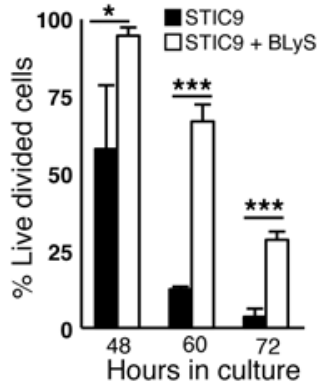

B C57BL/6
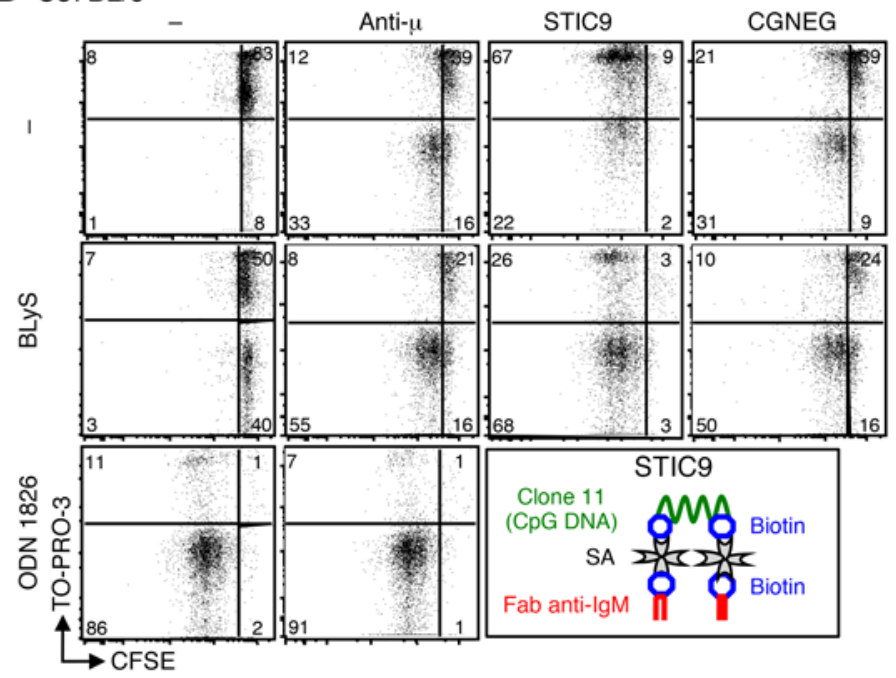

E

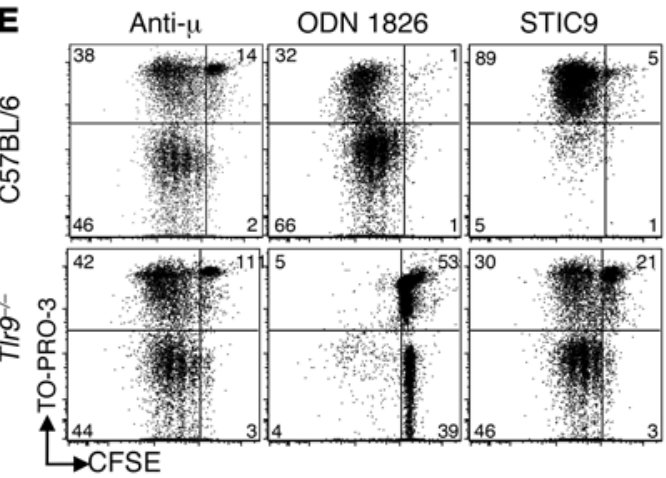

G

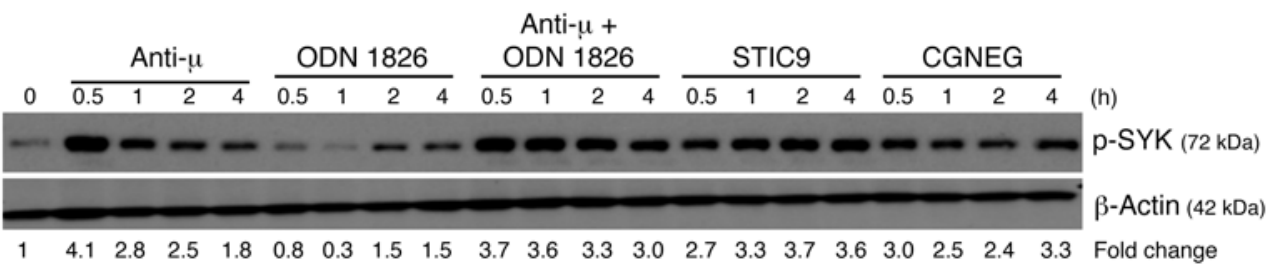

H

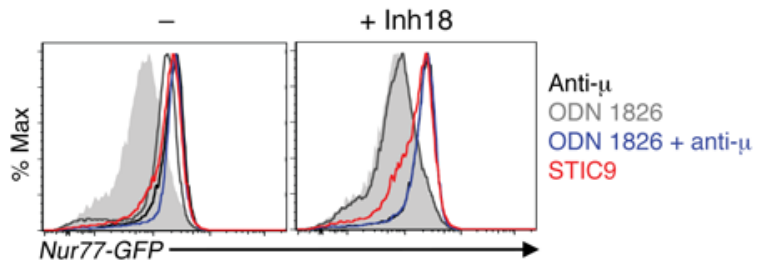

I Bio anti- $\mu-$ SA Bio ODN 1826

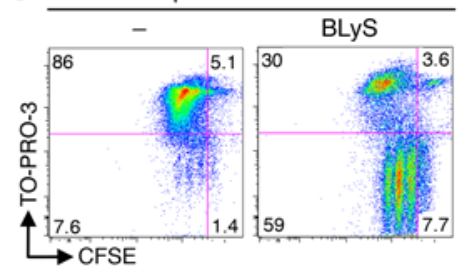

Figure 1. Addition of BLyS prevents AM14 and WT B cells from undergoing proliferation-associated cell death following stimulation with BCR-delivered TLR9 ligands. Representative FACS analysis at 60 hours (A) and percentage of live divided cells at 48, 60, and 72 hours (C) in AM14 CD23+ splenocytes cultured with the indicated stimuli in the presence or absence of BLyS. Dead cells were stained with TO-PRO-3, while CFSE dilution indicates proliferation. (B and D) Representative FACS analysis at 60 hours (B) and percentage of live divided cells at 48,60 , and 72 hours (D) in C57BL/6 CD23+ splenocytes cultured with the indicated stimuli in the presence or absence of BLyS. Diagram in B (inset) depicts the structure of STIC9. (E and F) Representative FACS analysis at 60 hours (E) and percentage of live divided cells 60 hours after STIC9 stimulation (F) in C57BL/6 and T/r $9^{-/-}$CD23 $3^{+}$B cells. (C) Immunoblot analysis of p-SYK in protein extracts isolated from CD23+ $557 \mathrm{BL} / 6$ splenocytes cultured with the indicated stimuli. Fold-change differences in expression are shown compared with unstimulated cells. Values in parentheses indicate the molecular weight. (H) FACS analysis of B cells from Nur77-GFP reporter mice at 5 hours, cultured with the indicated stimuli, with or without TLR9 inhibitor (Inh18) as described previously (84). Gray-filled area represents no stimulation; black line represents $F\left(a b^{\prime}\right)_{2}$ fragments of anti-IgM; gray line represents ODN 1826; blue line represents $F\left(a b^{\prime}\right)_{2}$ fragments of anti-IgM plus ODN 1826; and red line represents STIC9. Max, maximum. (I) Representative FACS plots show the proliferation and survival of C57BL/6 $\mathrm{CD}_{2} 3^{+}$splenocytes cultured for 60 hours with SA-linked biotinylated (Bio) ODN 1826 and biotinylated F(ab), in the presence or absence of BLyS. Data represent a minimum of 3 independent experiments with 3 mice each. Error bars indicate the mean $\pm \mathrm{SEM}$. ${ }^{*} P<0.05$ and ${ }^{* *} P<0.001$, by 2 -tailed Student's $t$ test. "-" signifies unstimulated cells. 

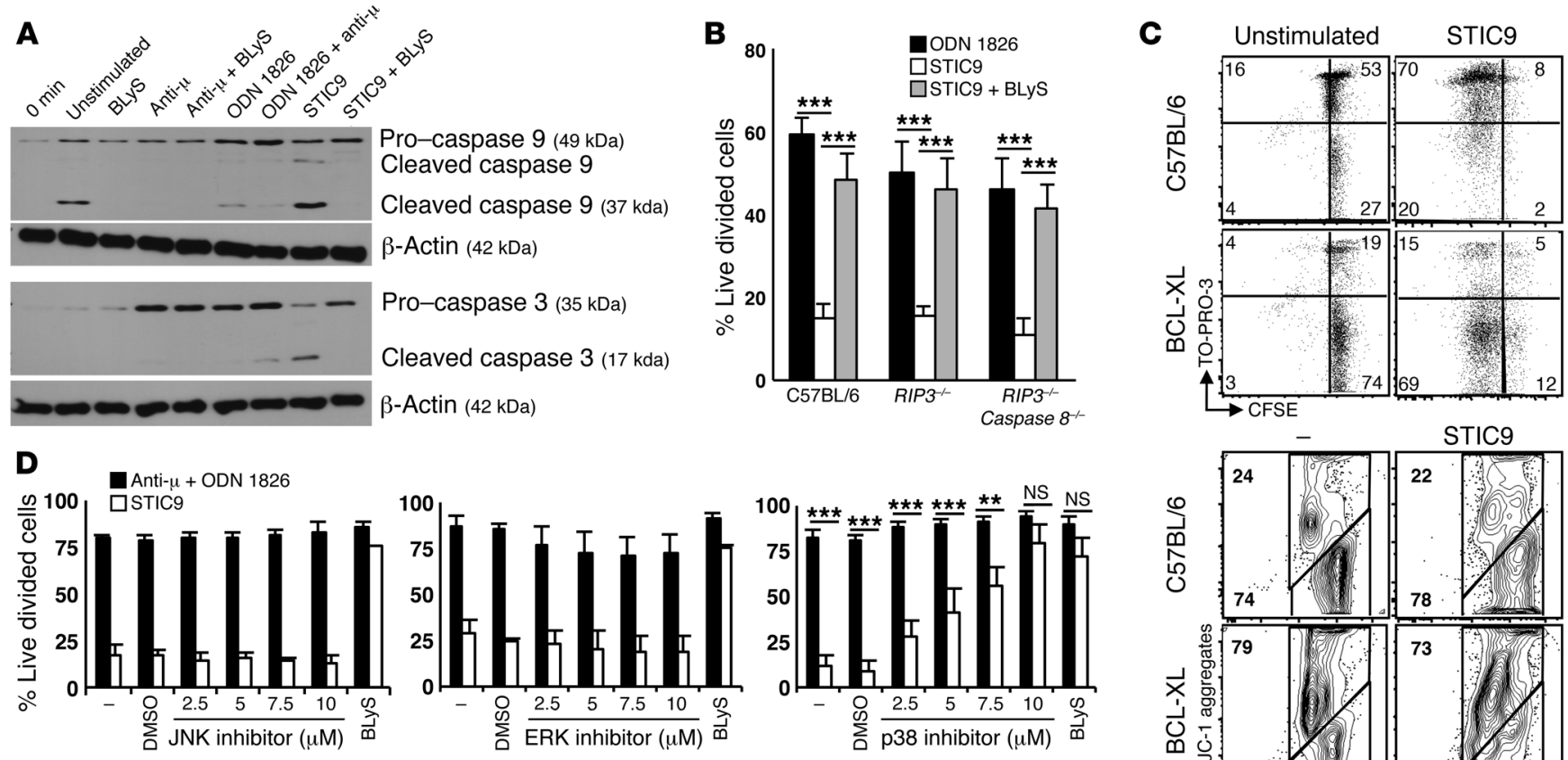

E
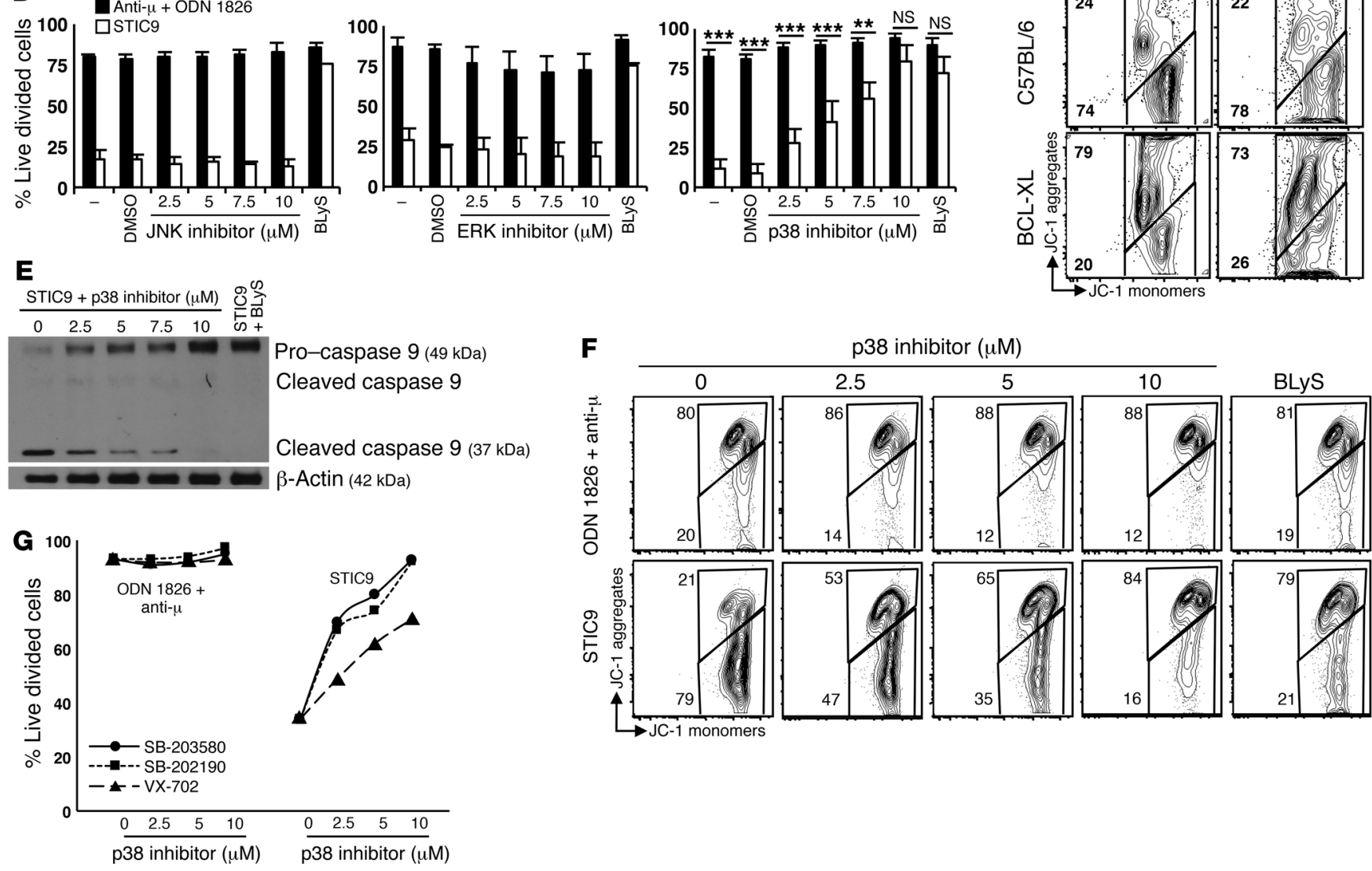

H

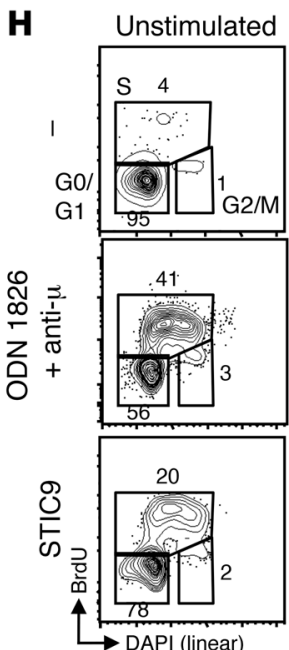

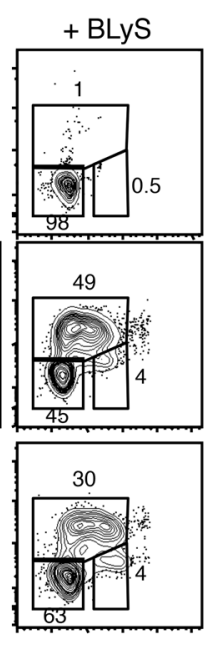

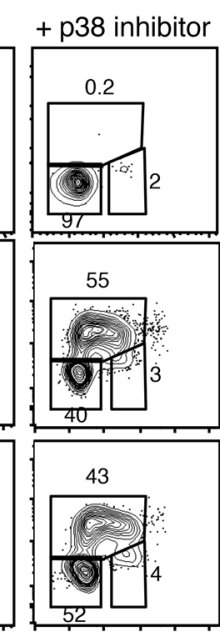

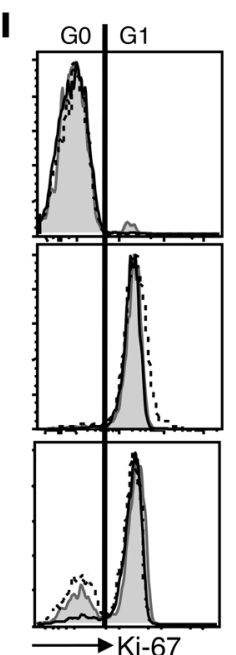

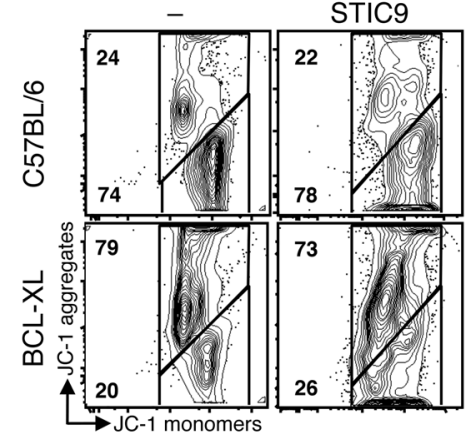


Figure 2. Cell death in response to STIC9 stimulation follows p38 MAPK-mediated cell-cycle arrest and mitochondrial apoptosis. (A) Immunoblot analysis of caspase 9 and caspase 3 cleavage in protein extracts from $\mathrm{CD} 23^{+} \mathrm{C} 57 \mathrm{BL} / 6$ splenocytes cultured for 60 hours with the indicated stimuli. Values in parentheses indicate the molecular weight.

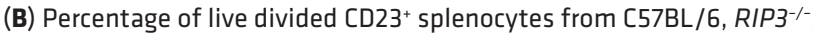
and $R I P 3^{-/-}$caspase $8^{-1-}$ mice following culture with the indicated stimuli. (C) Representative FACS plots of C57BL/6 and BCL-XL CD23+ splenocytes cultured for 60 hours with no stimulation or with STIC9 either loaded with CFSE and stained with TO-PRO-3 or stained with the mitochondrial stability-assessing dye JC-1. (D) Percentage of live divided C57BL/6 CD23 splenocytes following stimulation with either anti- $\mu$ or STIC9 in the presence of various concentrations of the JNK inhibitor SP600125, the MEK1/2 inhibitor U0126, or the p38 inhibitor SB203580. Since vehicle and non-vehicle control groups showed no differences, the latter was used for controls in subsequent experiments. (E) Immunoblot analysis of caspase 9 cleavage as described in A. (F) Representative FACS plots assessing the mitochondrial stability of $\mathrm{C} 57 \mathrm{BL} / 6 \mathrm{CD} 23^{+}$cells cultured for 60 hours with the indicated stimuli. (C) Percentage of live divided C57BL/6 CD23 ${ }^{+}$ splenocytes following culture as in $\mathbf{D}$ with various $\mathrm{p} 38$ inhibitors. ( $\mathbf{H}$ and $\mathbf{~}$ ) FACS analysis measuring the cell-cycle status of $\mathrm{C57BL} / 6 \mathrm{CD} 23^{+}$splenocytes cultured for 48 hours with the indicated stimuli. (I) $G_{0}$ and $G_{1}$ phases were distinguished through $\mathrm{Ki}-67$ staining. The gray area represents STIC9 alone; the dashed line represents STIC9 plus BLyS; and the solid black line represents STIC9 plus the p38 inhibitor SB203580. (J) Percentage of cells in the $S / G_{2} / M$ phase treated as in $\mathbf{H}$. (J and $\mathbf{K}$ ) FACS analysis measuring the cell-cycle status of BCL-XL-transgenic $\mathrm{CD}_{2} 3^{+}$splenocytes cultured for 48 hours. Error bars indicate the mean $\pm \mathrm{SEM} ; n \geq 3$ replicate analyses, and results are representative of 2 (B and $\mathbf{E}$ ) or a minimum of $3(\mathbf{A}, \mathbf{C}, \mathbf{D}$, and $\mathbf{F}-\mathbf{K}$ ) independent experiments. ${ }^{* *} P<0.005$ and ${ }^{* *} P<0.001$, by 2 -tailed Student's $t$ test. NS, not significant.

have been shown to affect B cell responses by modulating subcellular compartmentalization of TLR9 and MAPK signaling (49). Accordingly, we reasoned that STIC9 signals probably involve the MAPK pathways and asked whether inhibition of JNK, ERK, or p38 could block STIC9-induced apoptosis. Neither JNK nor ERK inhibition altered the patterns of response to ODN 1826 plus anti- $\mu$ or STIC9, despite both inducing the expected decrease in viability and proliferation with anti- $\mu$ (Figure 2D and Supplemental Figure 2E). In contrast, p38 inhibition abrogated STIC9induced cell death, and at $10 \mu \mathrm{M}$, the inhibitor rescued cells to the same extent as did BLyS (Figure 2D). Further, p38 inhibition prevented the caspase 9 cleavage and mitochondrial depolarization that otherwise follow STIC9 stimulation (Figure 2, E and F). To exclude off-target effects, we used 2 additional p38 inhibitors - SB202190 and VX702 - and both rescued STIC9-driven death (Figure 2G). Thus, BCR-delivered TLR9 ligands, unlike independent BCR or TLR9 stimulation, trigger a p38-dependent mechanism that leads to intrinsic mitochondrial apoptosis.

Following activation, B cell survival requires successful cellcycle transit, and p38 has been implicated in stress-induced cellcycle arrest (50). To determine the effect of STIC9 on cell-cycle status, B cells were stimulated and harvested after 48 hours, a time point at which viability in all cultures is comparable (Figure 1D). As expected, $44 \%$ of B cells stimulated with ODN 1826 plus anti- $\mu$ were in the $\mathrm{S} / \mathrm{G}_{2} / \mathrm{M}$ phase. In contrast, the majority of cells stimulated by STIC 9 were in $G_{1}$, with only $22 \%$ of the cells in S/ $G_{2} / M$ (Figure 2, $\mathrm{H}-\mathrm{J}$ ), in spite of the fact that most underwent several rounds of division (Figure 1B). Together, these findings suggest that STIC9stimulated cells experience cell-cycle arrest at the $\mathrm{G}_{1}-\mathrm{S}$ transition.
The addition of a p38 inhibitor reduced the proportion of cells in $G_{1}$, with a corresponding elevation of the proportion of cells in $\mathrm{S} /$ $\mathrm{G}_{2} / \mathrm{M}$ (Figure 2, H-J), suggesting that STIC9 stimulation induces $\mathrm{G}_{1} / \mathrm{S}$ cell-cycle arrest prior to mitochondrial apoptosis. However, it remained possible that cells undergoing mitochondrial apoptosis simply accumulate in the $G_{1}$ phase of the cell cycle. To address this possibility, we cultured B cells from BCL-XL mice with STIC9. Like C57BL/6 B cells, the majority of STIC9-stimulated BCL-XL transgene-positive cells were in $G_{0} / G_{1}$, despite their resistance to apoptosis (Figure 2K), consistent with the view that cell-cycle arrest precedes initiation of the intrinsic mitochondrial death pathway. Overall, these findings show that BCR-delivered TLR9 agonists terminate B cell activation through a p38-dependent cell-cycle arrest mechanism that subsequently drives mitochondrial cell death.

All preimmune B cell subsets undergo TLR9-dependent postproliferative death that can be rescued by BLyS. Both transitional (TR) and marginal zone (MZ) B cells express TLR9, include autoreactive or polyreactive clonotypes, and have been implicated in humoral autoimmune disease (51-53). Therefore, to examine how BCR-delivered TLR9 ligands affect these B cell subsets, we FACS sorted FO, MZ, TR type 2/3 (TR2/3), and TR type 1 (TR1) $\mathrm{B}$ cells as previously defined $(54,55)$. In accord with prior studies, anti- $\mu$ induced death among TR and MZ B cells (Figure 3A). In contrast, all subsets divided following stimulation with ODN 1826 plus anti- $\mu$ and were alive after 60 hours in culture. Mirroring our results with magnetic cell-sorting-enriched (MACS-enriched) $\mathrm{CD} 23^{+} \mathrm{B}$ cells, all subsets examined had proliferated following STIC9 stimulation and then died by 60 hours in culture (Figure $3 \mathrm{~A})$. Cell death in all subsets was most likely via mitochondrial intrinsic apoptosis, as cleavage of caspase 9 was observed in sorted FO, MZ, TR2/3, and TR1 B cells following STIC9 stimulation (Figure 3B). These data provide a previously unappreciated intrinsic role for TLR9 in limiting the responses of all preimmune B cell subsets to DNA-containing antigens.

BLyS allows STIC9-stimulated B cells to become antibody-secreting cells. Since B cells can be rescued from post-proliferative death by BLyS, we asked whether this or other signals enable progression to either antibody secretion or other differentiative fates. Accordingly, we first asked whether B cells stimulated with STIC9 and kept alive by BLyS could become antibody-secreting cells. As expected, $\mathrm{CD} 23^{+}$ $B$ cells cultured with BLyS alone secreted minimal antibody, however, both TR and FO subsets secreted 10-fold more antibody following stimulation with either ODN 1826 plus anti- $\mu$ or STIC9 plus BLyS (Table 1 and Figure 4A, upper panel). Supernatants from MZ $\mathrm{B}$ cells revealed a similar pattern of Ig secretion, albeit at a greater magnitude, consistent with their vigorous response to TLR stimuli and propensity to undergo rapid plasma cell differentiation (56). Moreover, enzyme-linked immunosorbent spot (ELISPOT) analyses confirmed that ODN 1826 plus anti- $\mu$ or STIC9 plus BLyS induced antibody-secreting cell (ASC) formation (Figure 4A, lower panel). Importantly, lambda ${ }^{+} \mathrm{B}$ cells from $3 \mathrm{H} 9 \mathrm{BCR}$-transgenic mice, which are dsDNA specific and normally eliminated at the TR stage (17, 57-60), were able to differentiate into ASCs following stimulation with STIC9 plus BLyS (Supplemental Figure 3C). These data reveal that TLR9 ligands delivered via the BCR yield a similar, self-limiting response by all major peripheral $\mathrm{B}$ cell subsets and that concomitant survival signals permit continued differentiation into ASCs. 

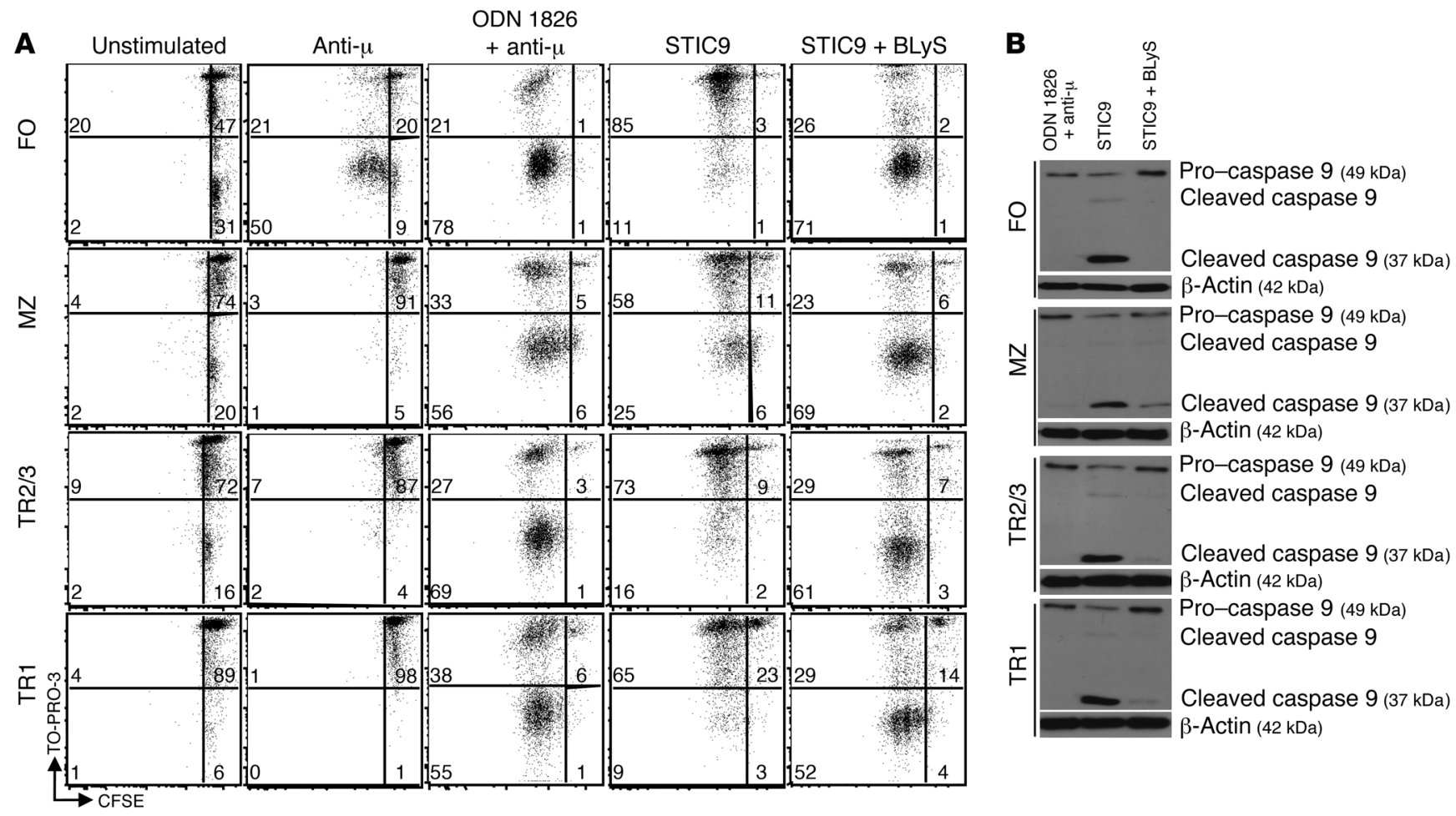

Figure 3. FO, MZ, and TR B cells behave similarly following STIC9 stimulation. (A) FACS analysis of proliferation and survival in sorted B220 $A A 4.1^{-}$ CD23+CD21/35- FO, B220 $A$ A4.1-CD23-CD21/35+ MZ, B220 $A A 4.1^{+} C D 23^{+}$TR2/3, and B220+AA4.1+CD23- TR1 splenic B cells cultured for 60 hours with no stimulation, with $F\left(a b^{\prime}\right)_{2}$ fragments of anti-IgM, with ODN 1826 plus $F\left(a b^{\prime}\right)_{2}$ fragments of anti-IgM, STIC9, or with STIC9 plus BLyS. Dead cells were stained by TO-PRO-3, while CFSE dilution indicates proliferation. (B) Immunoblot analysis of caspase 9 cleavage in protein extracts isolated from FO, MZ, TR2/3, and TR1 B cells cultured for 60 hours with ODN 1826 plus F(ab'), fragments of anti-IgM, STIC9, or STIC9 plus BLyS. Protein (10 $\mu$ g) was loaded into each well, and $\beta$-actin was used as a loading control. Values in parentheses indicate the molecular weight. All data are representative of 3 (A) or 2 (B) independent experiments.

CD40 costimulation and FO helper T cell cytokines rescue TLR9dependent post-proliferative death and foster a T-bet ${ }^{+}$fate. Although BLyS allows STIC9-stimulated B cells to survive and differentiate into ASCs, we reasoned that alternative second signals - such as CD40 costimulation and instructive cytokines characteristic of $\mathrm{T}$ cell-dependent immune responses - might foster alternative differentiative fates. Moreover, because T-bet expression and $\mathrm{IgG}_{2 \mathrm{a} / \mathrm{c}}$ isotype switching are regulated by IL-21, IFN- $\gamma$, and IL-4 in the context of TLR9 signals (35), we speculated that these features might extend to STIC9-stimulated cells. Accordingly, we assessed the effects of CD40 costimulation in the context of IL-21, IFN- $\gamma$, and IL- 4 on the survival and differentiative outcomes of STIC9-stimulated B cells.

CD40 signaling alone mirrored the findings with BLyS, rescuing the cells and enabling antibody secretion (Figure 4, A and B). The added presence of IFN- $\gamma$, IL-21, or IL- 4 did not affect CD40-mediated rescue (Figure 4B, upper panel). We reasoned that during $\mathrm{B}$ cell responses in vivo, CD40 signals would be delayed with respect to BCR-mediated activation, reflecting the need for $\mathrm{B}$ cell antigen processing and presentation to receive cognate $\mathrm{T}$ cell help. We therefore assessed rescue by CD 40 signals delivered 24 or 48 hours after STIC 9 stimulation. The results indicated that CD40 signals received within 24 hours of STIC9 stimulation yield equivalent rescue when these signals are received simultaneously and that even

48 hours after STIC9 stimulation, CD40 ligation affords partial rescue (Figure 4B, lower panel).

In accord with previous reports, both T-bet expression and IgG $G_{2 c}$ class switching increased markedly when either IFN- $\gamma$ or IL-21 (Figure 4, C-G) was added in conjunction with CD40 ligation; whereas, IL-4 fostered neither T-bet expression nor $\operatorname{IgG}_{2 \mathrm{c}}$ switching but instead yielded IgG $\mathrm{G}_{1}$ production. As shown previously in the context of TLR9 signaling (35), the induction of T-bet

\section{Table 1. Antibody production by cells cultured with STIC9 and BLyS}

Splenic B cell subset

FO

$\mathrm{MZ}$

TR2/3

TR1

$\begin{array}{ccc} & \text { Culture conditions } & \\ \text { BLyS } & \text { ODN 1826 + anti- } \mu & \text { STIC9 + BLyS } \\ 4 \pm 1 & 113 \pm 27^{\mathrm{B}} & 66 \pm 25^{\mathrm{A}} \\ 20 \pm 4 & 2,905 \pm 2,392 & 4,230 \pm 336^{\mathrm{B}} \\ 10 \pm 5 & 199 \pm 45^{\mathrm{B}} & 134 \pm 45^{\mathrm{B}} \\ 11 \pm 3 & 159.3 \pm 40^{\mathrm{B}} & 167 \pm 44^{\mathrm{B}}\end{array}$

Mean \pm SD of total lg $(\mathrm{ng} / \mathrm{ml})$ in supernatants from sorted subsets cultured for 60 hours. $n=3$ independent experiments. A 2-tailed Student's $t$ test was used for the statistical analysis. ${ }^{A}$ Denotes significant differences at $P<0.05$ for stimulation with BLyS alone. ${ }^{B}$ Denotes significant differences at $P<0.01$ for stimulation with BLyS alone. 


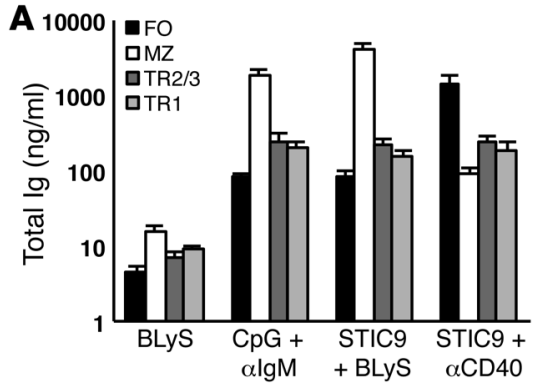

B
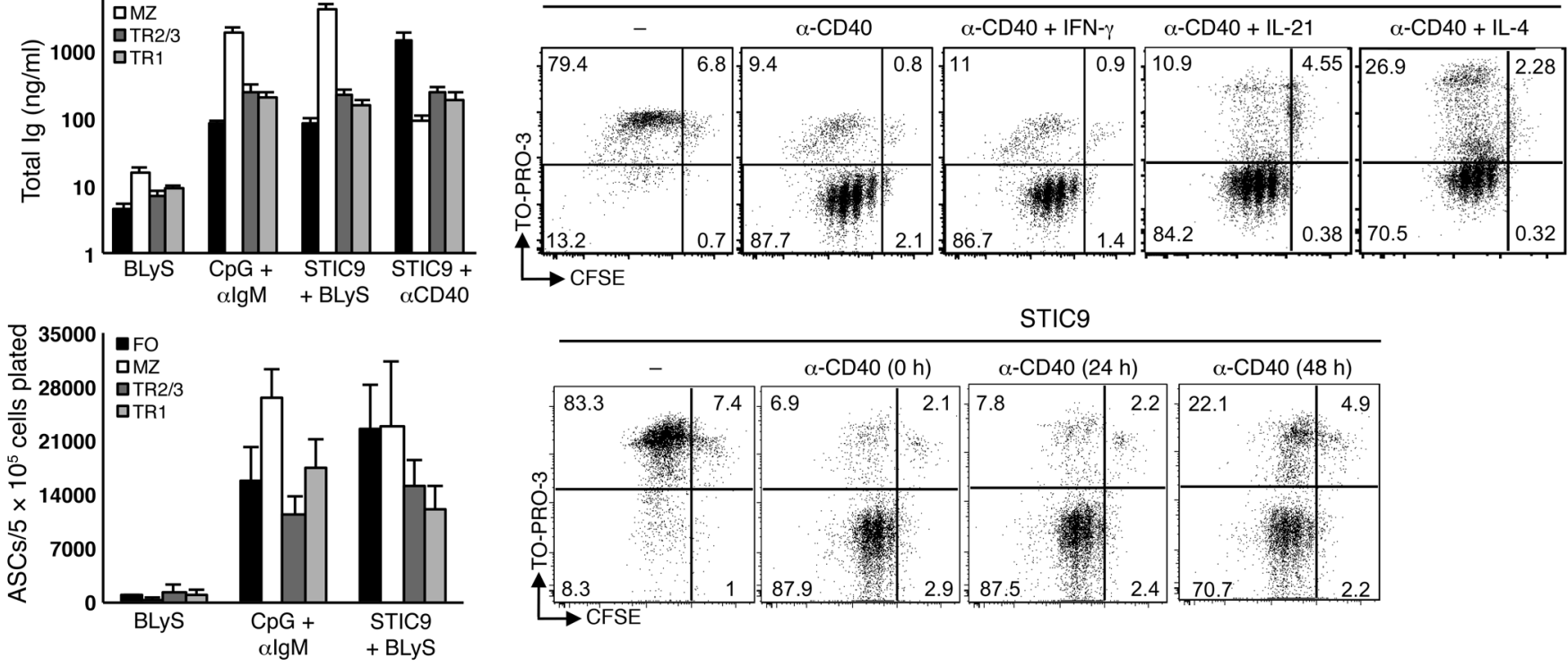

STIC9
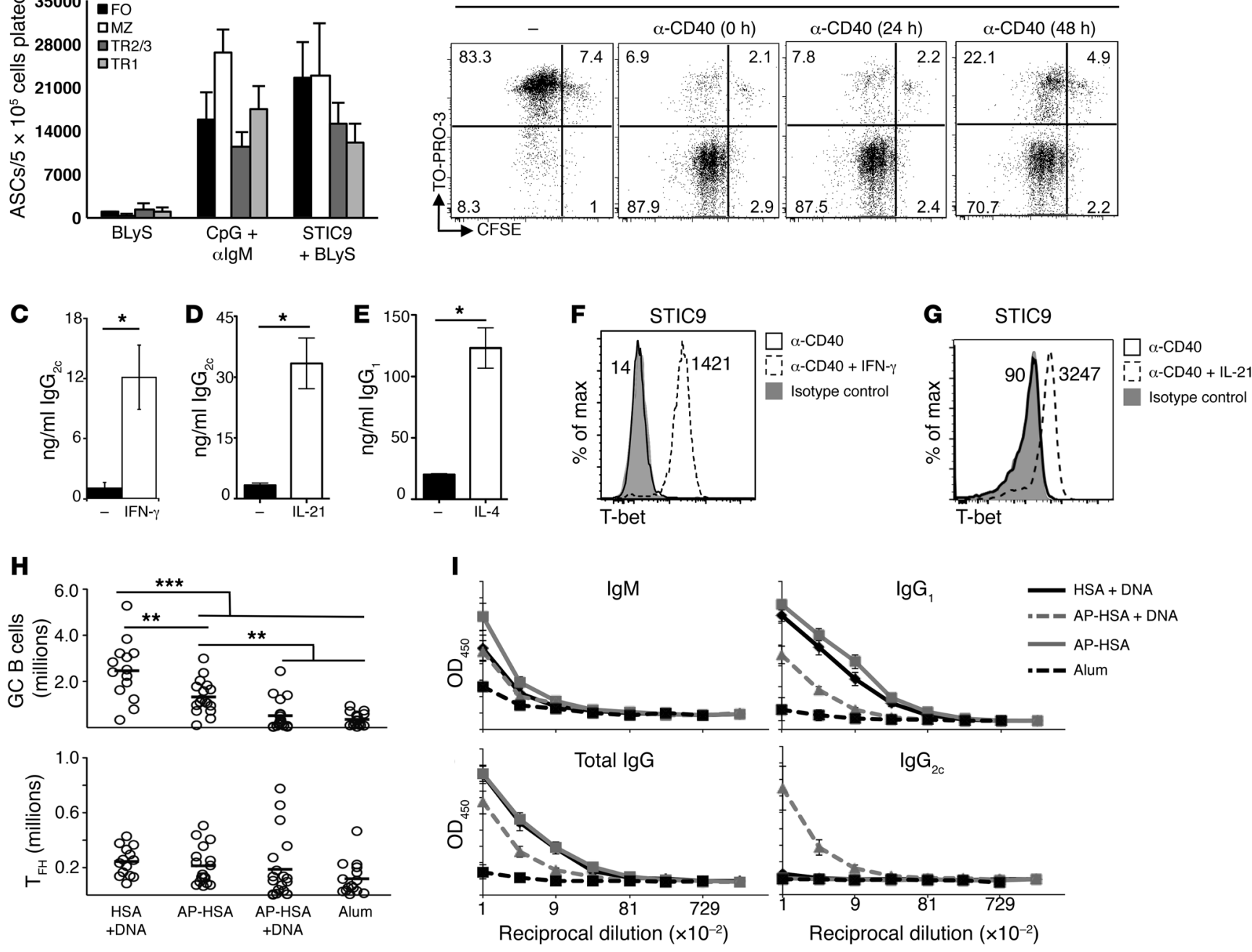

Figure 4. CD40 and Tfh cytokines promote survival, T-bet expression, and $\operatorname{lgC}_{2 \mathrm{c}}$ class-switching in STIC9-stimulated cells, and DNA conjugation modulates T cell-dependent B cell responses in vivo. (A) Sort-purified FO, MZ, TR2/3, and TR1 B cells cultured for 60 hours with the indicated stimuli. Total Ig was measured in the supernatants by ELISA, and total ASCs were measured by ELISPOT. Each stimulation group (CpC plus anti-IgM, STIC9 plus BLyS, and STIC9 plus anti-CD40) induced significantly more $(P<0.05)$ total Ig or ASCs compared with BLyS stimulation alone when compared with the respective $B$ cell subsets. (B) FACS analysis of the proliferation and survival of FO B cells cultured for 60 hours with the indicated cytokines and anti-CD40 added simultaneously with STIC9 stimulation (upper panels), or with anti-CD40 added at different time points after STIC9 stimulation (lower panels). (C-E) FO B cells were cultured with STIC9 plus anti-CD40, with or without (C) IFN- $\gamma$, (D) IL-21, or (E) IL-4 for 60 hours, following which (C and D) IgG ${ }_{2 c}$ and (E) IgC1 were measured by ELISA. (F and G) FO B cells were cultured with STIC9 plus anti-CD40, with or without (F) IFN- $\gamma$ or (C) IL-21 for 60 hours, and cells were probed for T-bet by intracellular flow staining. Plots show T-bet expression in live cells. Numbers inside the plots indicate the $\Delta$ mean fluorescence intensity (MFI)

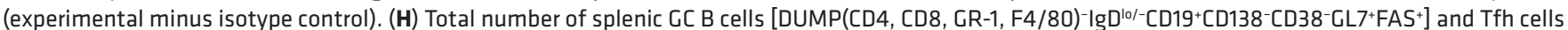

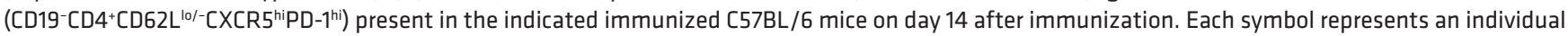
mouse. ANOVA with Bonferroni's correction was used for multiple comparisons. (I) HSA-specific serum titer in the indicated immunized mice on day 14 after immunization. All data are representative of 2 (A) or $3(\mathbf{B}-\mathbf{I})$ independent experiments. (C-E) Error bars indicate the mean $\pm \mathrm{SEM}$. ${ }^{*} P<0.05$,

${ }^{* *} P<0.005$, and ${ }^{* * *} P<0.001$, by 2 -tailed Student's $t$ test. 
A Unstimulated
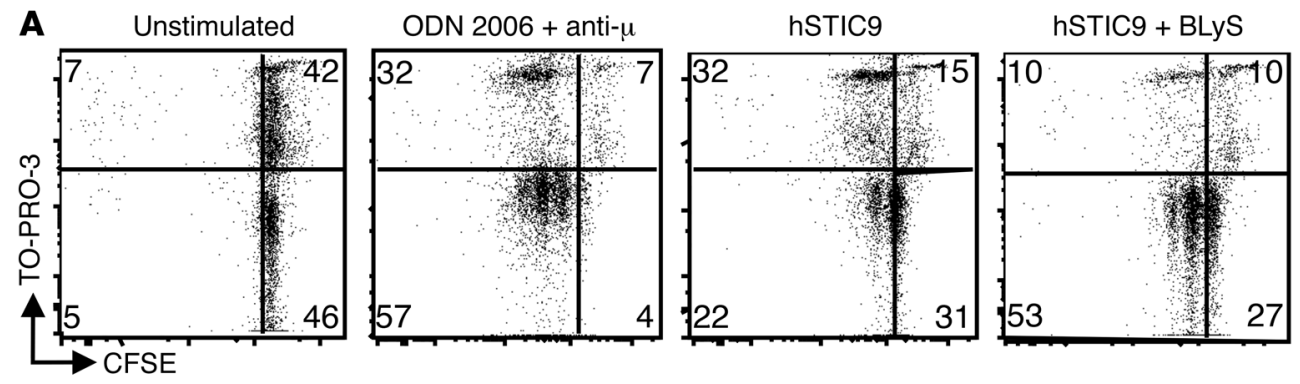

hSTIC9 + p38 inhibitor
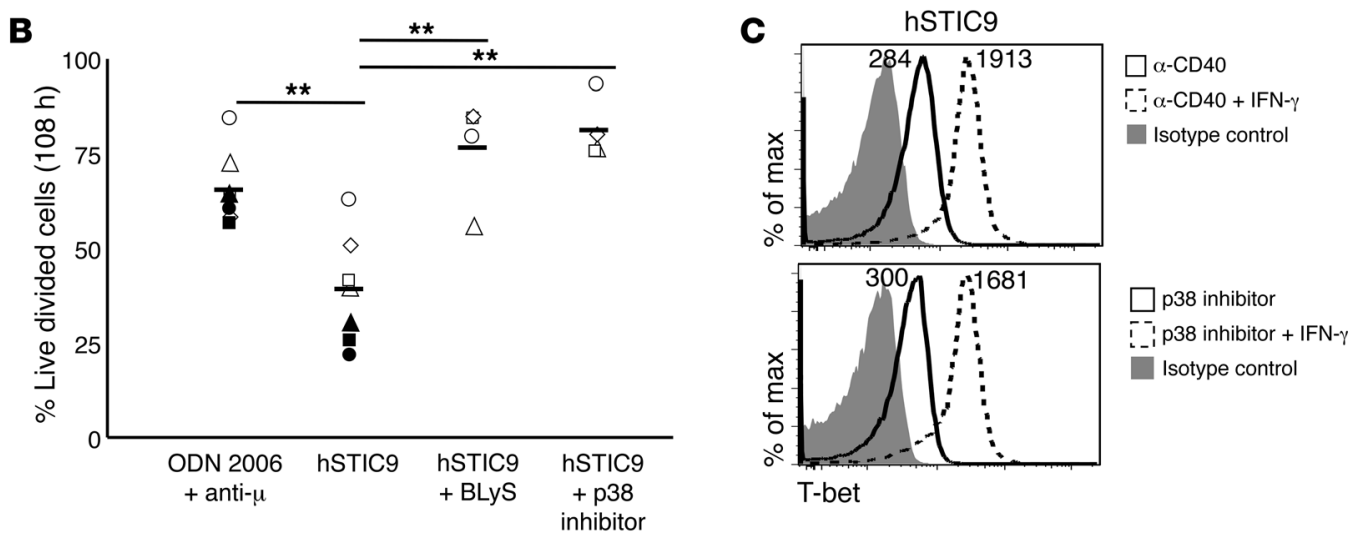

Figure 5. Naive human B cells undergo post-proliferation apoptosis following stimulation with BCR-internalized TLR9 ligands. (A) Representative FACS plots showing proliferation and survival in human CD27-CD19+ PBMCs cultured for 108 hours with no stimulation, $0 D N 2006$ plus F(ab') ${ }_{2}$ fragments of anti-IgM, hSTIC9, hSTIC9 plus BLyS, or hSTIC9 plus SB203580. Dead cells were stained by TO-PRO-3, while CFSE dilution indicates proliferation. (B) Percentage of live divided cells from multiple donors treated as in A. Each symbol represents a single donor. Black symbols $(n=3)$ indicate cells that were cultured with ODN 2006 plus F $\left(a b^{\prime}\right)_{2}$ fragments of anti-IgM and hSTIC9, while white symbols $(n=4)$ indicate cells that were additionally cultured with hSTIC9 plus BLyS or hSTIC9 plus the p38 inhibitor SB203580. (C) Human CD27-CD19+ PBMCs were cultured with STIC9 plus p38 inhibitor (10 $\mu$ M) or STIC9 plus anti-CD40, with or without IFN- $\gamma$, for 108 hours. At the end of the culture, cells were stained for T-bet. Plots show T-bet expression in live cells. Numbers inside the plot indicate $\Delta$ MFI (experimental minus isotype control). $n=3$ for all data, and results are representative of at least 3 independent experiments. ${ }^{* *} P<0.005$, by 2 -tailed Student's $t$ test.

expression by IFN- $\gamma$ and IL-21 occurs within the first 24 hours of activation and is independent of the extent to which $\mathrm{B}$ cells have divided after STIC9 plus anti-CD40 stimulation (Supplemental Figure 3, A and B).

Antigen-complexed DNA yields blunted humoral responses with $T$-bet ${ }^{+}$B cell-associated characteristics. Considered together, these in vitro analyses predicted that TLR9 agonists delivered by the BCR should limit B cell responses. We reasoned that in the absence of additional signals, incipient responses to DNA-containing antigens should prematurely terminate, whereas the presence of additional signals such as cognate CD4 help and cytokines should modulate the response and determine its quality. We used immunization with amyloid proteins to test this hypothesis in vivo. These misfolded proteins form fibrous structures termed amyloid aggregates, sometimes including cofactors such as DNA (61), and we have previously reported a method to produce amyloid proteins with or without DNA $(61,62)$. Using this approach, we immunized mice with amyloid precursor HSA (AP-HSA), AP-HSA linked to DNA, or native HSA mixed with, but not cross-linked to, DNA. Alum was used as an adjuvant in all conditions, and we also included an adjuvantonly control. On day 14 after immunization, mice receiving HSA plus DNA or AP-HSA had mounted an immune response with germinal center (GC) B cells, FO helper T cells (Tfh) (Figure 4H), and IgG $_{1}$ antibody (Figure 4I). In contrast, immunization with AP-HSA plus DNA yielded reduced GC B cell numbers that were equivalent to those detected with adjuvant only, as well as reduced titers of class-switched HSA-specific antibodies that were skewed toward $\operatorname{IgG}_{2 \mathrm{c}}$ (Figure 4, $\mathrm{H}$ and I). Interestingly, Tfh numbers were normal with this immunization (Figure $4 \mathrm{H}$ ). Immunization with AP-HSA plus DNA ICs yielded a reduction in GC B cell numbers, but not complete ablation of the response. This finding is in agreement with our in vitro observations (Figure $4, \mathrm{~A}$ and $\mathrm{B}$ ) suggesting that CD40 ligation affords rescue even several days after B cell activation by BCR ligands that contain a TLR9 agonist. Together, these findings are consistent with the overall relationships established by our in vitro analyses, since complexed DNA modulates the humoral response and, in the presence of cognate help, engenders the $\operatorname{IgG}_{2 \mathrm{c}}$ isotype-switching characteristic of T-bet induction.

$B C R$-delivered TLR9 ligands limit human CD27 B cell responses. Finally, we questioned whether human B cells are similarly regulated by DNA-containing antigens. Human TLR9 signals optimally to a different CpG motif than does murine TLR9, but this motif appears in the clone 11 sequence that we used in STIC9. Therefore, we designed a human stimulatory TLR9 complex (hSTIC9) by linking biotinylated clone 11 to biotinylated Fab anti-human IgM with SA. Naive (CD27-CD19+) human B cells were isolated and cultured for 108 hours, when proliferative responses most closely resembled those seen after 60 hours with murine CD23+ $\mathrm{B}$ cells (Figure 
$5 \mathrm{~A})$. As expected, the majority of human B cells cultured with ODN 2006 plus $\mathrm{F}\left(\mathrm{ab}^{\prime}\right)_{2}$ anti-human IgM were alive and had proliferated (Figure 5, A and B). Mirroring the response of murine B cells, human B cells stimulated with hSTIC9 underwent a proliferative burst followed by apoptosis, and either BLyS addition or p38 inhibition rescued this death (Figure 5, A and B). Moreover, when human $B$ cells were rescued from death, they expressed T-bet, which was further enhanced in the presence of IFN- $\gamma$ (Figure $5 \mathrm{C}$ ). These findings strongly suggest that human and murine preimmune B cells undergo similar responses to BCR-delivered TLR9 ligands and that these probably reflect similar molecular mechanisms.

\section{Discussion}

This study reveals a relationship among BCR, TLR9, and cytokine signals that regulate $\mathrm{B}$ cell responses to DNA-containing antigens. BCR ligands that deliver a TLR9 agonist yield a brief proliferation that is terminated by cell-cycle arrest and intrinsic apoptotic death. This self-limiting response is characteristic of all major preimmune B cell subsets in mice and humans and requires MAPK p38 activation. BLyS prolongs this otherwise short-lived response, enabling the activated B cells to survive and differentiate into ASCs, whereas CD40 costimulation and Tfh cytokines yield both antibody secretion and differentiation into the autoimmuneassociated T-bet ${ }^{+} \mathrm{B}$ cell phenotype. Together, these observations disclose a mechanism that prevents or limits responses to DNAassociated BCR ligands and may help explain how TLR9 deficiency promotes humoral autoimmunity.

The proapoptotic role of TLR9 shown here is seemingly at odds with an extensive literature showing that TLR9 agonists engender $\mathrm{B}$ cell division without cell death and are effective vaccine adjuvants (63). However, TLR9 signaling in these contexts likely differs from the BCR-mediated internalization of natural ICs or STIC9, as such small molecules do not involve BCR engagement in their uptake $(64,65)$. In addition, TLRs can signal from a range of endolysosomal compartments with varying functional outcomes $(66,67)$. Studies of simultaneous engagement of BCR and TLR9, similar to STIC9, showed that BCR and TLR9 colocalize in autophagosomes and initiate the hyperphosphorylation of p38 MAPK after such activation. Results of these studies also demonstrated the unique signaling pathways by which $\mathrm{B}$ cells regulate responses to DNA-containing antigens, namely, by governing the subcellular location of TLR9 and MAPK signaling $(49,68)$. Whether free CpG ODNs reach the same compartment as BCR-delivered TLR9 ligands is unclear, but CpG ODN and ICs elicit different cytokines from AM14 B cells (48), consistent with fundamentally different signaling outcomes. Further, while the affinity of TLR9 for DNA components of different ligands is a potentially confounding factor, ODN 1826 engenders post-proliferative death when conjugated to $\mathrm{F}\left(\mathrm{ab} \mathrm{b}_{2}{ }_{2}\right.$ anti- $\mu$, favoring the interpretation that BCR-mediated delivery is the key distinction. Similarly, BCR engagement and signal strength might also impact outcome. However, multiple measures of BCR signal strength indicate that BCR signals induced by each reagent used herein are comparable and further support the notion that BCR-associated delivery of the TLR9 agonist underlies this unique response.

The STIC9 reagent affords the separation of BCR-mediated proliferative signals from TLR9-dependent cell death, thus enabling interrogation of the downstream pathways. While one might argue that a dominant, TLR9-mediated death pathway is involved, this seems unlikely, because simultaneous but independent ligation of TLR9 and the BCR does not result in cell death. Instead, we favor the notion that crosstalk between these systems is responsible, whereby TLR9 ligation impedes or redirects survival signals that otherwise accompany BCR signaling. The exact points of intersection remain unclear, but engagement of both the BCR and TLR9 initiates the NF- $\mathrm{BB}$ and MAPK pathways, so crosstalk between these, including the documented hyperactivation of p38 (49), likely plays a role in the post-proliferative death mechanism reported herein. Indeed, our findings reveal a central role for p38 in STIC9-induced cell-cycle arrest and mitochondrial apoptosis, functions that have previously been associated with the p38 pathway (69-71). Cell-cycle arrest and mitochondrial apoptosis may reflect parallel but independent consequences of p38 signaling following stimulation with BCR-delivered TLR9 ligands. Alternatively, as cell-cycle arrest occurs even when apoptosis is blocked, it may be a proximal event that leads directly to mitochondrial destabilization. Regardless, both reflect potential limiting mechanisms that prevent continued expansion or prolonged survival of autoreactive B cell clones.

The TLR9-mediated post-proliferative death response that we observed is likely relevant to the etiology of humoral autoimmunity. For example, it may help explain why TLR9-deficient autoimmune strains develop more severe disease than do their TLR9-sufficient counterparts $(15,16,18-20)$. Consistent with this notion, autoimmune disease is similarly exacerbated when TLR9 deficiency is limited to B cells (72). Nevertheless, in addition to its negative regulatory role, TLR9 is also required for B cell activation, ASC differentiation, and the production of isotype-switched, DNA-reactive autoantibodies in autoimmune-prone mice $(15,32)$. Further, this unique response - an initial proliferative burst followed by rapid elimination - is common to all major preimmune subsets in mice and humans. This suggests that TR and MZ B cells, which contain polyreactive and autoreactive clones, could be recruited to clear TLR9-containing BCR ligands such as apoptotic debris, but are quickly eliminated to avoid sustained activity against self-components. Alternatively, this mechanism might be important for purging self-reactive B cells at the immature and TR stages. In fact, in 3H9 mice, either exogenous BLyS or TLR9 deficiency extends the lifespan of dsDNA-specific B cells normally lost at the TR stage $(17,59)$, and mutations impacting TLR9 function are associated with a more polyreactive B cell repertoire (73). These possibilities are not mutually exclusive and could both serve as checkpoints in the prevention of autoimmune disease.

Impeding the TLR9-mediated post-proliferative death response through either intrinsic failure in the relevant pathways or misdirected survival and differentiation signals could increase the propensity for autoimmune pathogenesis, and it is tempting to speculate that these differences might lead to distinct risk and disease features. For example, circumventing this mechanism through excess BLyS might afford the formation of short-lived autoreactive plasmablasts. Indeed, BLyS depletion therapy has had mixed success in ameliorating systemic lupus erythematosus (SLE) flares, perhaps reflecting restoration of this pathway in a subset of patients (24). In contrast to BLyS, T cell-mediated rescue of BCR-TLR9 coengagement might 
foster GC differentiation, allowing somatic hypermutation, affinity maturation, and the generation of long-lived plasma or memory B cells. This is supported by our observations that addition of antiCD40 with the IL-21 and IFN- $\gamma$ cytokines engenders survival and induces T-bet expression and class-switching to $\operatorname{IgG}_{2 \mathrm{a} / \mathrm{c}}$. Since T-bet ${ }^{+}$ $B$ cells have been reported in the context of aging, autoimmunity, and infections (74), these cells may arise in response to TLR9 ligands delivered via the BCR, and while such signals would ordinarily trigger apoptosis, cognate $\mathrm{T}$ cell help and additional cytokines could result in the breakthrough of autoantibody production. Additionally, in vivo and in vitro data suggest that RNA-sensing receptors, such as TLR7, do not promote post-proliferative cell death, but instead foster plasma cell differentiation (75). Thus, activation via these pathways may require concomitant TLR9 signals to control the overall response $(30,33,76)$. Indeed, in mouse models in which TLR7 plays a critical role, TLR9 haploinsufficiency exacerbates disease $(15,16$, 18-20). Finally, some B cell subsets may resist this mechanism. Our preliminary observations indicate that $\mathrm{CD} 27^{+}$human B cells may resist hSTIC9-driven post-proliferative death, suggesting that some memory B cell subsets are refractory. Thus, TLR9 may play a unique role in limiting the duration of potentially autoreactive responses, and alternative routes for bypassing this regulatory system may underlie some of the variability in the clinical features and therapeutic outcomes observed in humoral autoimmunity.

\section{Methods}

Mice. C57BL/6 and Nur77-GFP reporter mice (77) were purchased from the The Jackson Laboratory. AM14 mice were maintained at the University of Massachusetts. Tlr $9^{-/}$mice were provided by $\mathrm{P}$. Scott (University of Pennsylvania). Taci-/ $(78)$, 3H9-transgenic, and B cell-specific BCL-XL-transgenic (79) mice were maintained at the University of Pennsylvania. BR3-deficient mice were maintained at the University of Southern California (Los Angeles, California, USA). Caspase $8^{-/-} \mathrm{RIP3}^{-/-}$and $\mathrm{RIP3}^{-/-}$mice were provided by William Kaiser and Edward Mocarski (Emory University, Atlanta, Georgia, USA).

B cell cultures. B cells were collected from 8- to 16-week-old male and female mice. Splenic B cells were isolated by positive selection and cultured as described previously $(38,39,44,76,80)$. Briefly, B cells were stimulated with 10 micrograms $/ \mathrm{ml} \mathrm{F}(\mathrm{ab})_{2}{ }_{2}$ fragments of goat antiIgM (Jackson ImmunoResearch Laboratories); $1 \mu \mathrm{g} / \mathrm{ml}$ anti-CD40 (clone HM40-3; BD); and $1 \mu \mathrm{M} \mathrm{CpG} \mathrm{DNA} \mathrm{(ODN} \mathrm{1826;} \mathrm{InvivoGen).}$ STIC9 ICs were formed by combining a biotinylated CG-rich dsDNA fragment (39) with SA and Fab anti-mouse IgM at a final concentration of $0.5 \mu \mathrm{g} / \mathrm{ml} \mathrm{dsDNA}, 0.13 \mu \mathrm{g} / \mathrm{ml} \mathrm{SA}$, and $0.5 \mu \mathrm{g} / \mathrm{ml} \mathrm{Fab}$ anti-mouse IgM. Murine IL-21, IL-4, and IFN- $\gamma$ (Shenandoah Biotechnology Inc.) were used at 25, 10, and $10 \mathrm{ng} / \mathrm{ml}$, respectively. Human peripheral blood mononuclear cells (PBMCs) were isolated through negative selection with magnetic anti-human CD27 beads, followed by positive selection with anti-human CD19 beads (Miltenyi Biotec) and cultured in round-bottomed plates for 108 hours. Cells were loaded with CFSE (Invitrogen, Thermo Fisher Scientific) as described previously (44). Human PBMCs were collected from both healthy men and women, aged 28-52 years. Inhibitor studies used 2.5-10 $\mu \mathrm{M}$ SB203580 (InvivoGen); SB202190 (Cell Signaling Technology); VX-702 (Cayman Chemical); U0126 (Cell Signaling Technology); or SP600125 (InvivoGen). Stimulations had $500 \mathrm{ng} / \mathrm{ml}$ recombinant human BLyS (rhBLyS) (Human Genome Sciences Inc. or R\&D Systems); $10 \mu \mathrm{g} /$ $\mathrm{ml} \mathrm{F}\left(\mathrm{ab}^{\prime}\right)_{2}$ anti-human IgM (Jackson ImmunoResearch Laboratories); $1 \mu \mathrm{M}$ CpG DNA (ODN 2006; InvivoGen); or hSTIC9, formed by combining $1 \mu \mathrm{g} / \mathrm{ml}$ biotinylated clone 11 DNA; $0.5 \mu \mathrm{g} / \mathrm{ml} \mathrm{SA}$ (New England Biolabs); and $0.25 \mu \mathrm{g} / \mathrm{ml} \mathrm{Fab}$ anti-human IgM, $\mathrm{FC}_{5 \mu}$ fragment specific (Jackson ImmunoResearch Laboratories) for 1 hour at $4^{\circ} \mathrm{C}$.

Flow cytometry. For all analyses, live/dead discrimination was assessed using either a LIVE/DEAD Fixable Aqua Stain Kit, DAPI, or TO-PRO-3 (Invitrogen, Thermo Fisher Scientific). Spleens were disrupted to single-cell suspensions and red blood cells lysed using ammonium-chloride-potassium (ACK) buffer (Lonza). Fluorochromeconjugated or biotinylated antibodies against mouse CD19 (clone 6D5), B220 (clone RA3-6B2), CD21/CD35 (clone CR2/CR1), AA4.1/ CD93 (clone AA4.1), IgD (clone 11-26c.2a), CD138 (clone 281-2), CXCR5 (clone L138D7), PD-1 (clone RMP1-30), TCR $\beta$ (clone H57597), and T-bet (clone 4B10) were purchased from BioLegend; CD23 (clone B3B4), IgM (clone R6-60.2), CD95 (clone Jo2), and CD62L (clone MEL-14) from BD Biosciences; and CD4 (clone RM4-5), CD8 (clone 53-6.7), F4/80 (clone BM8), GR-1 (clone RB6-8C5), and CD38 (clone 90) from eBioscience. Cells were stained with antibodies in $\mathrm{PBS} / 1 \%$ BSA containing mouse IgG Fc fragments (Jackson ImmunoResearch Laboratories). Mouse IgG1, $\kappa$ antibody (clone MOPC21; BioLegend) was used as an isotype control for T-bet staining, as described previously (35). BR3 and TACI were detected with antimouse BR3 (clone eBio7H22-E16; eBioscience) or anti-mouse TACI (clone 8F10; R\&D Systems), with rat IgG1, $\kappa$ antibody (clone eBRG1; eBioscience) and rat IgG2a, $\kappa$ antibody (clone RTK2758; R\&D Systems), respectively, as isotype controls. All stains were incubated for 30 minutes at $4^{\circ} \mathrm{C}$, except for CXCR5, which was added first and incubated at room temperature for 1 hour. Staining with biotinylated antibodies was followed by staining with Brilliant Violet 650-conjugated SA (BioLegend). Data were collected on a BD LSR II Flow Cytometer and analyzed with FlowJo software (Tree Star).

Mitochondrial membrane potential. Mitochondrial membrane potential was analyzed by staining with JC-1 (BD Biosciences) according to the manufacturer's protocol.

Cell-cycle analysis. Cells were pulsed with $50 \mu \mathrm{g} / \mathrm{ml} \mathrm{BrdU}$ for the last 90 minutes of cell culture; fixed and permeabilized using BD Cytofix Solutions A and B (BD Bioscience); incubated with $225 \mu \mathrm{g} / \mathrm{ml}$ DNase solution in $150 \mathrm{mM} \mathrm{NaCl}$ and $400 \mathrm{mM} \mathrm{MgCl}_{2}$ for 35 minutes at room temperature; and stained with FITC anti-BrdU antibody (B44; BD Bioscience) and DAPI. $G_{0} / G_{1}$ was differentiated with anti-Ki-67 (16A8; BioLegend).

Immunoblot analysis. Lysates were prepared and separated by SDS-PAGE as described previously $(81,82)$. Proteins were quantified by a Bio-Rad assay (Bio-Rad). Protein $(10 \mu \mathrm{g})$ was loaded into each well, and $\beta$-actin was used as a loading control. Immunoblots were performed using rabbit anti-mouse caspase 9, rabbit anti-mouse caspase 3 , or rabbit anti-mouse phosphorylated SYK (p-SYK) (all from Cell Signaling Technology). Peroxidase-conjugated donkey anti-rabbit IgG $(\mathrm{H}+\mathrm{L})$ (Jackson ImmunoResearch) was used as a secondary detection antibody. Peroxidase-conjugated $\mathrm{mAb}$ against mouse $\beta$-actin (AC-15; Sigma-Aldrich) was used as a loading control. Quantity One 1-D Analysis Software (Bio-Rad) was used for gel densitometry.

ELISA and ELISPOT analyses. Plates were coated with $10 \mu \mathrm{g} /$ $\mathrm{ml}$ anti-Ig $(\mathrm{H}+\mathrm{L})$ (SouthernBiotech) as previously described (83). For ELISA, culture supernates were plated and incubated with secondary HRP-conjugated goat anti-mouse Igא plus anti-mouse $\operatorname{Ig} \lambda$ antibodies 
(SouthernBiotech). Plates were analyzed as previously described (83). For ELISPOTs, cells that had been cultured for 48 hours were replated and cultured for 8 hours at $37^{\circ} \mathrm{C}$, then developed with biotin-conjugated anti-mouse Igא plus anti-mouse Ig $\lambda$ (SouthernBiotech) as previously described (83). Plates (Corning) were coated overnight with $1 \mu \mathrm{g} / \mathrm{ml}$ native HSA and blocked for 2 hours in PBS/2\% BSA. Sera were added to the first row of plates at a 1:100 dilution, with serial 3-fold dilutions down the rows. Anti-HSA antibodies were detected with goat antimouse IgG, IgG $\operatorname{Ig}_{1}$, and IgM conjugated with HRP (SouthernBiotech). TMB Substrate Reagent (BD) was used to detect HRP activity, and $2 \mathrm{M}$ sulfuric acid (J.T. Baker) was used to stop the reaction. Plates were read at $\mathrm{OD}_{450}$ on an EMax Microplate Reader (Molecular Devices).

Preparation of amyloid and immunization. AP-HSA was prepared as previously described (62). Briefly, HSA (Sigma-Aldrich) was incubated for 2 hours in EDC (1-ethyl-3-[3-dimethyl-aminopropyl] carbodiimide hydrochloride) (Sigma-Aldrich) at a ratio of 1:5 (w/w). The mixture was neutralized by adding $10 \%(\mathrm{v} / \mathrm{v})$ of $1 \mathrm{M}$ Tris- $\mathrm{HCl}$ (Invitrogen, Thermo Fisher Scientific) at pH 10.5 and then dialyzed overnight into sterile PBS. To produce AP-HSA plus DNA, AP-HSA was incubated for more than 2 hours with E. coli 12 genomic DNA (InvivoGen) at a 7:3 $(w / w)$ ratio of protein to DNA. As a control for an immunogen containing unlinked DNA and protein, native HSA was mixed with DNA at a 7:3 (w/w) ratio. Each preparation was precipitated in aluminum potassium sulfate (Thermo Fisher Scientific) at $0.25 \mathrm{mg} / \mathrm{ml} \mathrm{10 \%} \mathrm{alum} \mathrm{solu-}$ tion. The $\mathrm{pH}$ was adjusted to 6.5 , and precipitates were resuspended in sterile PBS. Mice were immunized i.p. with $70 \mu$ g protein, and spleens and sera were collected upon sacrifice 14 days later.

Statistics. All data are represented as the mean \pm SEM. Statistical analysis was performed using a 2-tailed, equal-variance Student's $t$ test. ANOVA analysis with Bonferroni's correction was used for multigroup comparisons in Figure 4H. A P value of less than 0.05 was considered statistically significant.

Study approval. Mice were handled according to a protocol approved by the IACUC of the University of Pennsylvania and the Animal Care and Use Review Office of the US Army Medical Research and Materiel Command (AMRMC). Human samples were obtained from the University of Pennsylvania Human Immunology Core, which maintains an IRB-approved protocol for secondary use of deidentified human donor specimens. Use of these cells in this study did not constitute human subjects research as defined by the NIH, and exempt determinations were made by the IRB of the University of Pennsylvania and the US AMRMC Office of Research Protections, Human Research Protection Office.

\section{Author contributions}

VJS, MAO, KM, MN, LEH, LZ, NG, KN, AMS, SDE, and AM performed experiments; MPC, AMR, WS, WC, and TK helped design experiments and provided reagents; and MAO, VJS, MPC, and AMR wrote the manuscript.

\section{Acknowledgments}

We thank Eline Luning Prak and Andrew Wells (University of Pennsylvania, Philadelphia, PA) for their valuable technical advice. We also thank Chandra Mohan and Yong Du (University of Houston, Houston, TX) for providing spleens from Sle1, Sle2, and Sle 3 mice. This work was supported by the Office of the Assistant Secretary of Defense for Health Affairs, through the Peer Reviewed Medical Research Program (award nos. W81WWXWH-10-1-0185 and W81XWH-14-1-0305 to MPC). Opinions, interpretations, conclusions and recommendations are those of the authors and are not necessarily endorsed by the Department of Defense. This work was also supported by the National Institute of Arthritis and Musculoskeletal and Skin Diseases (NIAMS), NIH grant PO1 AR-050256 (to AMR); and NIH grant R01 AR-050193 (to WS). MAO was supported in part by NIH Training Grant T32 AI-055428. NG and KM were supported in part by NIH Training Grant T32 AI-07309. KM was supported in part by NIAMS, NIH grant F31 AR-063597.

Address correspondence to: Michael P. Cancro, Department of Pathology and Laboratory Medicine, University of Pennsylvania, Perelman School of Medicine, Philadelphia, Pennsylvania 191046082, USA. Phone: 215.898.8067; E-mail: cancro@mail.med. upenn.edu.
1. Erikson J, Radic MZ, Camper SA, Hardy RR, Carmack C, Weigert M. Expression of anti-DNA immunoglobulin transgenes in non-autoimmune mice. Nature. 1991;349(6307):331-334.

2. Nemazee DA, Bürki K. Clonal deletion of B lymphocytes in a transgenic mouse bearing anti-MHC class I antibody genes. Nature. 1989;337(6207):562-566.

3. Meffre E, Wardemann H. B-cell tolerance checkpoints in health and autoimmunity. Curr Opin Immunol. 2008;20(6):632-638.

4. Tiller T, Tsuiji M, Yurasov S, Velinzon K, Nussenzweig MC, Wardemann H. Autoreactivity in human $\operatorname{IgG}^{+}$memory B cells. Immunity. 2007;26(2):205-213.

5. Wardemann H, Yurasov S, Schaefer A, Young JW, Meffre E, Nussenzweig MC. Predominant autoantibody production by early human B cell precursors. Science. 2003;301(5638):1374-1377.

6. Hiepe F, Dörner T, Hauser AE, Hoyer BF, Mei $\mathrm{H}$, Radbruch A. Long-lived autoreactive plasma cells drive persistent autoimmune inflammation.
Nat Rev Rheumatol. 2011;7(3):170-178.

7. Zaheen A, Martin A. Activation-induced cytidine deaminase and aberrant germinal center selection in the development of humoral autoimmunities. Am J Pathol. 2011;178(2):462-471.

8. Diamond B, Katz JB, Paul E, Aranow C, Lustgarten $\mathrm{D}$, Scharff MD. The role of somatic mutation in the pathogenic anti-DNA response. Annu Rev Immunol. 1992;10:731-757.

9. Jiang C, et al. Abrogation of lupus nephritis in activation-induced deaminase-deficient MRL/ lpr mice. JImmunol. 2007;178(11):7422-7431.

10. Shlomchik M, et al. Anti-DNA antibodies from autoimmune mice arise by clonal expansion and somatic mutation. JExp Med. 1990;171(1):265-292.

11. Kotzin BL. Systemic lupus erythematosus. Cell. 1996;85(3):303-306.

12. Green NM, Marshak-Rothstein A. Toll-like receptor driven $\mathrm{B}$ cell activation in the induction of systemic autoimmunity. Semin Immunol. 2011;23(2):106-112.
13. Groom JR, et al. BAFF and MyD88 signals promote a lupuslike disease independent of T cells. JExp Med. 2007;204(8):1959-1971.

14. Mackay F, et al. Mice transgenic for BAFF develop lymphocytic disorders along with autoimmune manifestations. J Exp Med. 1999;190(11):1697-1710.

15. Christensen SR, Kashgarian M, Alexopoulou L, Flavell RA, Akira S, Shlomchik MJ. Tolllike receptor 9 controls anti-DNA autoantibody production in murine lupus. J Exp Med. 2005;202(2):321-331.

16. Christensen SR, Shupe J, Nickerson K, Kashgarian M, Flavell RA, Shlomchik MJ. Toll-like receptor 7 and TLR9 dictate autoantibody specificity and have opposing inflammatory and regulatory roles in a murine model of lupus. Immunity. 2006;25(3):417-428.

17. Nickerson KM, Christensen SR, Cullen JL, Meng W, Luning Prak ET, Shlomchik MJ. TLR9 promotes tolerance by restricting survival of anergic anti-DNA B cells, yet is also required for their 
activation. JImmunol. 2013;190(4):1447-1456.

18. Nickerson KM, et al. TLR9 regulates TLR7- and MyD88-dependent autoantibody production and disease in a murine model of lupus. J Immunol. 2010;184(4):1840-1848.

19. Santiago-Raber ML, et al. Critical role of TLR7 in the acceleration of systemic lupus erythematosus in TLR9-deficient mice. J Autoimmun. 2010;34(4):339-348

20. Yu P, et al. Toll-like receptor 9-independent aggravation of glomerulonephritis in a novel model of SLE. Int Immunol. 2006;18(8):1211-1219.

21. Cheema GS, Roschke V, Hilbert DM, Stohl W. Elevated serum B lymphocyte stimulator levels in patients with systemic immune-based rheumatic diseases. Arthritis Rheum. 2001;44(6):1313-1319.

22. Mariette X, et al. The level of BLyS (BAFF) correlates with the titre of autoantibodies in human Sjögren's syndrome. Ann Rheum Dis. 2003;62(2):168-171.

23. Sanz I, Yasothan U, Kirkpatrick P. Belimumab. Nat Rev Drug Discov. 2011;10(5):335-336.

24. Stohl W, Scholz JL, Cancro MP. Targeting BLyS in rheumatic disease: the sometimes-bumpy road from bench to bedside. Curr Opin Rheumatol. 2011;23(3):305-310.

25. Zhang J, et al. Cutting edge: a role for B lymphocyte stimulator in systemic lupus erythematosus. J Immunol. 2001;166(1):6-10.

26. Bubier JA, et al. A critical role for IL-21 receptor signaling in the pathogenesis of systemic lupus erythematosus in BXSB-Yaa mice. Proc Natl Acad Sci U S A. 2009;106(5):1518-1523.

27. Ettinger R, Kuchen S, Lipsky PE. The role of IL-21 in regulating $\mathrm{B}$-cell function in health and disease. Immunol Rev. 2008;223:60-86.

28. Hu X, Ivashkiv LB. Cross-regulation of signaling pathways by interferon-gamma: implications for immune responses and autoimmune diseases. Immunity. 2009;31(4):539-550.

29. Pollard KM, Cauvi DM, Toomey CB, Morris KV, Kono DH. Interferon- $\gamma$ and systemic autoimmunity. Discov Med. 2013;16(87):123-131.

30. Berland R, et al. Toll-like receptor 7-dependent loss of $\mathrm{B}$ cell tolerance in pathogenic autoantibody knockin mice. Immunity. 2006;25(3):429-440.

31. Kono DH, et al. Endosomal TLR signaling is required for anti-nucleic acid and rheumatoid factor autoantibodies in lupus. Proc Natl Acad Sci US A. 2009;106(29):12061-12066.

32. Lartigue A, et al. Role of TLR9 in anti-nucleosome and anti-DNA antibody production in lpr mutation-induced murine lupus. J Immunol. 2006;177(2):1349-1354.

33. Lee PY, et al. TLR7-dependent and FcgammaR-independent production of type I interferon in experimental mouse lupus. J Exp Med. 2008;205(13):2995-3006.

34. Silver KL, Crockford TL, Bouriez-Jones T, Milling S, Lambe T, Cornall RJ. MyD88-dependent autoimmune disease in Lyn-deficient mice. Eur J Immunol. 2007;37(10):2734-2743.

35. Naradikian MS, et al. Cutting edge: IL-4, IL-21, and IFN- $\gamma$ interact to govern T-bet and CD11c expression in TLR-activated B cells. JImmunol. 2016;197(4):1023-1028.

36. Rubtsov AV, et al. Toll-like receptor 7 (TLR7)driven accumulation of a novel CD11 $\mathrm{c}^{+} \mathrm{B}$-cell population is important for the development of autoimmunity. Blood. 2011;118(5):1305-1315.

37. Rubtsov AV, Rubtsova K, Kappler JW, Marrack P. TLR7 drives accumulation of ABCs and autoantibody production in autoimmune-prone mice. Immunol Res. 2013;55(1-3):210-216.

38. Leadbetter EA, Rifkin IR, Hohlbaum AM, Beaudette BC, Shlomchik MJ, Marshak-Rothstein A. Chromatin-IgG complexes activate B cells by dual engagement of IgM and Toll-like receptors. Nature. 2002;416(6881):603-607.

39. Uccellini MB, et al. Autoreactive B cells discriminate $\mathrm{CpG}$-rich and $\mathrm{CpG}$-poor DNA and this response is modulated by IFN-alpha. JImmunol. 2008;181(9):5875-5884.

40. Kaiser WJ, et al. RIP3 mediates the embryonic lethality of caspase-8-deficient mice. Nature. 2011;471(7338):368-372.

41. Bossen C, Schneider P. BAFF, APRIL and their receptors: structure, function and signaling. Semin Immunol. 2006;18(5):263-275.

42. Hatada EN, et al. NF-kappa B1 p50 is required for BLyS attenuation of apoptosis but dispensable for processing of NF-kappa B2 p100 to p52 in quiescent mature B cells. JImmunol. 2003;171(2):761-768.

43. Hsu BL, Harless SM, Lindsley RC, Hilbert DM, Cancro MP. Cutting edge: BLyS enables survival of transitional and mature B cells through distinct mediators. J Immunol. 2002;168(12):5993-5996.

44. Treml LS, et al. TLR stimulation modifies BLyS receptor expression in follicular and marginal zone B cells. JImmunol. 2007;178(12):7531-7539.

45. Harless SM, et al. Competition for BLySmediated signaling through $\mathrm{Bcm}$ /BR3 regulates peripheral B lymphocyte numbers. Curr Biol. 2001;11(24):1986-1989.

46. Li X, Jiang S, Tapping RI. Toll-like receptor signaling in cell proliferation and survival. Cytokine. 2010;49(1):1-9.

47. Dal Porto JM, Gauld SB, Merrell KT, Mills D, PughBernard AE, Cambier J. B cell antigen receptor signaling 101. Mol Immunol. 2004;41(6-7):599-613.

48. Busconi L, et al. Functional outcome of B cell activation by chromatin immune complex engagement of the B cell receptor and TLR9. J Immunol. 2007;179(11):7397-7405.

49. Chaturvedi A, Dorward D, Pierce SK. The B cell receptor governs the subcellular location of Toll-like receptor 9 leading to hyperresponses to DNA-containing antigens. Immunity. 2008;28(6):799-809.

50. Ambrosino C, Nebreda AR. Cell cycle regulation by p38 MAP kinases. Biol Cell. 2001;93(1-2):47-51.

51. Lopes-Carvalho T, Kearney JF. Marginal zone B cell physiology and disease. Curr Dir Autoimmun. 2005;8:91-123.

52. Wardemann H, Yurasov S, Schaefer A, Young JW, Meffre E, Nussenzweig MC. Predominant autoantibody production by early human B cell precursors. Science. 2003;301(5638):1374-1377.

53. Chen X, Martin F, Forbush KA, Perlmutter RM Kearney JF. Evidence for selection of a population of multi-reactive B cells into the splenic marginal zone. Int Immunol. 1997;9(1):27-41.

54. Hardy RR, Li YS, Allman D, Asano M, Gui M, Hayakawa K. B-cell commitment, development and selection. Immunol Rev. 2000;175:23-32.
55. Cancro MP. Peripheral B-cell maturation: the intersection of selection and homeostasis. Immunol Rev. 2004;197:89-101.

56. Meyer-Bahlburg A, Rawlings DJ. Differential impact of Toll-like receptor signaling on distinct B cell subpopulations. Front Biosci (Landmark Ed). 2012;17:1499-1516

57. Erikson J, Radic MZ, Camper SA, Hardy RR, Carmack C, Weigert M. Expression of anti-DNA immunoglobulin transgenes in non-autoimmune mice. Nature. 1991;349(6307):331-334.

58. Gay D, Saunders T, Camper S, Weigert M. Receptor editing: an approach by autoreactive B cells to escape tolerance. J Exp Med. 1993;177(4):999-1008.

59. Hondowicz BD, et al. The role of BLyS/BLyS receptors in anti-chromatin $\mathrm{B}$ cell regulation. Int Immunol. 2007;19(4):465-475.

60. Radic MZ, Erikson J, Litwin S, Weigert M. B lymphocytes may escape tolerance by revising their antigen receptors. J Exp Med. 1993;177(4):1165-1173.

61. Di Domizio J, et al. Binding with nucleic acids or glycosaminoglycans converts soluble protein oligomers to amyloid. J Biol Chem. 2012;287(1):736-747.

62. Dorta-Estremera SM, Li J, Cao W. Rapid generation of amyloid from native proteins in vitro. $\mathrm{J}$ Vis Exp. 2013;5(82):50869.

63. Wagner $\mathrm{H}$. The immunogenicity of $\mathrm{CpG}$ antigen conjugates. Adv Drug Deliv Rev. 2009;61(3):243-247.

64. Lahoud MH, et al. DEC-205 is a cell surface receptor for CpG oligonucleotides. Proc Nat Acad Sci US A. 2012;109(40):16270-16275.

65. Roberts TL, et al. B cells do not take up bacterial DNA: an essential role for antigen in exposure of DNA to toll-like receptor-9. Immunol Cell Biol. 2011;89(4):517-525.

66. Guiducci C, et al. Properties regulating the nature of the plasmacytoid dendritic cell response to Toll-like receptor 9 activation. J Exp Med. 2006;203(8):1999-2008.

67. Sasai M, Linehan MM, Iwasaki A. Bifurcation of Toll-like receptor 9 signaling by adaptor protein 3. Science. 2010;329(5998):1530-1534.

68. Chaturvedi A, Pierce SK. How location governs toll-like receptor signaling. Traffic. 2009;10(6):621-628.

69. De Chiara G, et al. Bcl-2 Phosphorylation by 38 MAPK: identification of target sites and biologic consequences. J Biol Chem. 2006;281(30):21353-21361.

70. Cai B, Chang SH, Becker EB, Bonni A, Xia Z. p38 MAP kinase mediates apoptosis through phosphorylation of BimEL at Ser-65. J Biol Chem. 2006;281(35):25215-25222.

71. Thornton TM, Rincon M. Non-classical p38 map kinase functions: cell cycle checkpoints and survival. Int J Biol Sci. 2009;5(1):44-51.

72. Jackson SW, et al. Opposing impact of B cell-intrinsic TLR7 and TLR9 signals on autoantibody repertoire and systemic inflammation. J Immunol. 2014;192(10):4525-4532.

73. Isnardi I, et al. IRAK-4- and MyD88-dependent pathways are essential for the removal of developing autoreactive B cells in humans. Immunity. 2008;29(5):746-757. 
74. Rubtsova K, Rubtsov AV, Cancro MP, Marrack P. Age-associated B cells: A T-bet-dependent effector with roles in protective and pathogenic immunity. J Immunol. 2015;195(5):1933-1937.

75. Nündel K, et al. Cell-intrinsic expression of TLR9 in autoreactive B cells constrains BCR/ TLR7-dependent responses. JImmunol. 2015;194(6):2504-2512.

76. Green NM, Moody KS, Debatis M, Marshak-Rothstein A. Activation of autoreactive B cells by endogenous TLR7 and TLR3 RNA ligands. J Biol Chem. 2012;287(47):39789-39799.

77. Zikherman J, Parameswaran R, Weiss A. Endogenous antigen tunes the responsive- ness of naive B cells but not T cells. Nature. 2012;489(7414):160-164.

78. von Bülow GU, van Deursen JM, Bram RJ. Regulation of the T-independent humoral response by TACI. Immunity. 2001;14(5):573-582.

79. Grillot DA, et al. bcl-x exhibits regulated expression during $\mathrm{B}$ cell development and activation and modulates lymphocyte survival in transgenic mice. J Exp Med. 1996;183(2):381-391.

80. Goenka R, et al. Local BLyS production by $\mathrm{T}$ follicular cells mediates retention of high affinity $\mathrm{B}$ cells during affinity maturation. J Exp Med. 2014;211(1):45-56.

81. Schmidt AM, et al. Diacylglycerol kinase $\zeta$ limits the generation of natural regulatory T cells. Sci Signal. 2013;6(303):ra101.

82. Stadanlick JE, et al. Tonic B cell antigen receptor signals supply an NF-kappaB substrate for prosurvival BLyS signaling. Nat Immunol. 2008;9(12):1379-1387.

83. Oropallo MA, et al. Chronic spinal cord injury impairs primary antibody responses but spares existing humoral immunity in mice. JImmunol. 2012;188(11):5257-5266.

84. Avalos AM, Uccellini MB, Lenert P, Viglianti GA, Marshak-Rothstein A. Fc $\gamma$ RIIB regulation of BCR/ TLR-dependent autoreactive B-cell responses. Eur JImmunol. 2010;40(10):2692-2698. 